\title{
IMAGING BIOLOGICAL TISSUES BY UTILIZING ULTRASOUND AND ELECTROMAGNETIC FIELDS
}

\author{
by \\ ELENA RENZHIGLOVA \\ B.Sc. (hons.), Physics, \\ Rostov State University, Russia, \\ 2005 \\ A thesis \\ presented to Ryerson University \\ in partial fulfillment of the \\ requirements for the degree \\ of Master of Science \\ in the program of \\ BIOMEDICAL PHYSICS
}

Toronto, Ontario, Canada, 2009

(C)Elena Renzhiglova 2009 


\section{Author's Declaration}

I hereby declare that I am the sole author of this thesis or dissertation.

I authorize Ryerson University to lend this thesis or dissertation to other institutions or individual s for the purpose of scholarly research.

I further authorize Ryerson University to reproduce this thesis or dissertation by photocopying or by other means, in total or in part, at the request of other institutions or individuals for the purpose of scholarly research. 


\section{Abstract \\ Elena Renzhiglova}

Imaging Biological Tissues by Utilizing Ultrasound and Electromagnetic Fields

$$
\text { MSc., Biomedical Physics }
$$

Ryerson University, Toronto, 2009

This thesis reports our research on developing a new method to image the electric conductivity and relative permittivity of biological tissues. The first method is Differential Frequency Magneto-Acousto-Electrical Tomography (DF-MAET) to image the electrical impedance of biological tissues with high spatial resolution. It is shown that DF-MAET signal is caused by the vibrations of the sample at a difference frequency (DF) because of the radiation force. In the second method, we investigated the possibility of using a novel mechanism for imaging the electrical permittivity of biological tissues. Theoretical study shows that a magnetic moment will be produced in biological tissues when both an ultrasound wave and an electrical field exist in the tissue. We report the results to detect this magnetic moment with both coils and electrodes attached to the tissue. We were able to detect the signal with electrodes, but its frequency dependence indicates that this signal is due to the impedance modulation by ultrasound, and that it is not related to the relative permittivity. Finally, we studied the ultrasonic vibration potentials generated in fat and muscle tissues. 


\section{Acknowledgements}

I would like to express my sincere appreciation of help and support provided by following organizations and individuals:

Ryerson University and the Department of Physics;

My supervisor Dr. Yuan Xu;

Members of Research Committee Dr. J. Tavakkoli and Dr. Michael Joy (Department of Electrical Engineering, University of Toronto);

A. Worthington and B. Soroushian;

My course instructors K. Cumaradas, M. Kolios, A. Pejovic-Milic, P. Goldman, V. Toronov;

Fellow graduate and undergraduate students, especially S. Haider, V. Ivantsiv, A. Hrbek, S. Azish and O. Doganay for cooperation in research and E. da Silva and B. Holder for the help with preparation of the paper draft and this thesis;

My friends who believed in me and motivated me. 


\section{Contents}

1 Introduction 2

1.1 Basics of bioimpedance . . . . . . . . . . . . . . . . . 2

1.1.1 Electrical properties of tissues and their relation to different pathologies 2

1.1.2 Composition of biological tissues . . . . . . . . . . . . 3

1.1.3 Frequency dispersion of permittivity of biological tissues . . . . . 7

1.1.4 Electrical properties of tissues and cells ........... 8

1.1.5 Electrical properties of cancer tissues . . . . . . . . . . . . . 11

1.2 Imaging electrical properties of tissues f . . . . . . . . . . . . 12

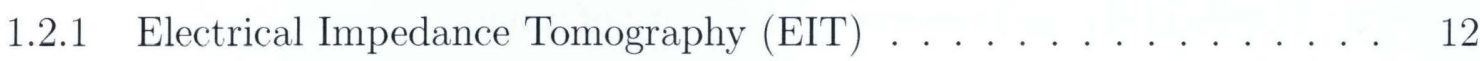

1.2 .2 Magnetic Induction Tomography _. . . . . . . . . . . . 15

1.2.3 Magnetic Resonance Electrical Impedance Tomography (MREIT) . . 17

1.3 Thesis objectives and outline . . . . . . . . . . . . . . . . . 19

2 Differential frequency Magneto-Acousto-Electrical Tomography (DF-MAET) 20

2.0.1 DF-MAET signal induced by radiation force . . . . . . . . . 23

2.1 Methods and experimental setup . . . . . . . . . . . . . . . 29

2.2 Characterization of the acoustic field . . . . . . . . . . . 30 
2.3 DF-MAET experimental results . . . . . . . . . . . . . . . . . . . . 32

2.3.1 Dependence of the amplitude of the DF-MAET signal on the modulation frequency $\ldots \ldots \ldots \ldots \ldots \ldots \ldots$

2.3.2 Spatial resolution in DF-MAET . . . . . . . . . . . . 36

2.3.3 Dependence of the DF-MAET signal on the acoustical properties of the sample . . . . . . . . . . . . . . .

2.3.4 Parametric effect in the medium . . . . . . . . . . . . . . . 42

2.4 Discussion . . . . . . . . . . . . . . . . . . . . . . . . . . . . . . . 43

2.5 Conclusion . . . . . . . . . . . . . . . . . . . . . . 46

3 The possibility of imaging permittivity of biological tissues with ultrasound

3.1 Magnetic moment induced by applying ultrasound to a polarized tissue . . . 47

3.1 .1 Principle . . . . . . . . . . . . . . . . . . 47

3.1.2 Experimental setup and results: detection with coil . . . . . . . . 51

3.2 Conductivity and relative permittivity of a muscle tissue . . . . . . . . 52

3.3 Detection of the magnetic moment with electrodes . . . . . . . . . . . 58

3.3.1 Sample and experimental setup . . . . . . . . . . . . . . 58

3.3 .2 Results . . . . . . . . . . . . . . . . . . . . . 6 6 60

3.4 Discussion. Acousto-electric interaction signals . . . . . . . . . . . . 66

4 Contrast provided by ultrasonic vibration potentials $\quad 69$

4.1 Experiments with heterogeneous samples . . . . . . . . . . . . . . . 69

4.2 Electrode signal from pork muscle at frequency of $10 \mathrm{MHz} \ldots \ldots . \ldots 72$ 
4.3 Discussion. Debye effect in electrolytes and physiological liquids . . . . . . . 76

5 Overall conclusions and future work

5.1 Future work .......................... . . . 80

A Electrical, magnetic and acoustic properties of the media and materials used in experiments

$\begin{array}{lr}\text { B Publications and conference presentations } & 84\end{array}$ 


\section{List of Figures}

1.1 Illustration of the metabolism between the blood and the biological tissues. . 5

1.2 Composition of an animal cell. . . . . . . . . . . . . . 6

1.3 Composition of a lipid bi-layer membrane. . . . . . . . . . . . . 7

1.4 Typical frequency dependence of the relative permittivity $\epsilon_{r}$ and conductivity $\sigma$ of a biological tissue $[1] \ldots \ldots \ldots \ldots$

1.5 Electrical model of a cell with nucleus. . . . . . . . . . . . . . . . 10

1.6 Distribution of the injected current in the cell at a frequency below (a) and above (b) the characteristic frequency of $\beta$-dispersion. . . . . . . . . . 11

1.7 Single (a) and multiple (b) source EIT systems. . . . . . . . . . . . . . . 13

1.8 Phasor diagram representing the primary $(\mathbf{B})$ and the secondary $(\Delta \mathbf{B})$ magnetic fields in MIT. . . . . . . . . . . . . . . . . . . . . 16

2.1 Illustration of lead field current density distribution in a sample. . . . . . . . 21

2.2 MAET experimental setup. . . . . . . . . . . . . . . . . . . . 21

2.3 An experiment to illustrate the cancellation of the signal from the homogenous interior. A conductive gelatin sample with oil-filled cavity (a), and signals when the beam was focused at the points A (b) and B (c) are shown [1]. . . 
2.4 The 2D image of the pressure distribution of a $2.25 \mathrm{MHz}$ transducer in the focal zone (a) and the line plot across the dashed line (b) . . . . . . . . 31

$2.54 \mathrm{kHz}$ component of the signal from the sample (solid line) and empty space (dashed line). . . . . . . . . . . . . . . . . . .

2.6 Spectrum of DF-MAET signal (a) and its fit with the power function $y=b x^{a}(b) 34$

2.7 Normalized amplitudes of hydrophone acoustic and DF-MAET signals plotted against modulation frequency to show the correlation between them when $\delta f$ is larger than $6.4 \mathrm{kHz} . \ldots \ldots \ldots \ldots \ldots$

2.8 Diagram of the phantom to study the resolution in the DF-MAET. . . . . 37

2.9 Edge-spread (a) and line-spread functions (b) of the DF-MAET imaging system for $f_{m}=1 \mathrm{kHz} \ldots \ldots \ldots \ldots \ldots \ldots \ldots \ldots$

2.10 Edge-spread (a) and line-spread functions (b) of the differential MAET imaging system for $f_{m}=43 \mathrm{kHz} \ldots \ldots \ldots \ldots \ldots . \ldots \ldots$

2.11 Illustration for the experiments described in section 3.3 to study the effect of the mechanical properties of the sample on the nonlinear MAET signal. . . .

$2.121 \mathrm{~mm}$ scan of a gelatine with air bubble (a) and vertical amplitude plot along

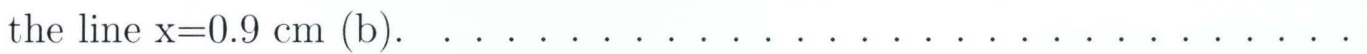

2.13 Vertical scans of the sample bridge at different distances away from transducer. 42

3.1 Schematic of the setup to detect the signal from a biological tissue through

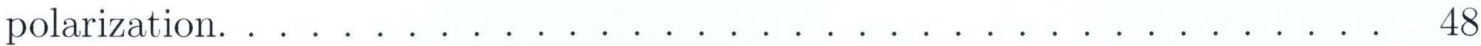

3.2 Schematic representation of a pick-up coil connected to amplifier. . . . . . . 51

3.3 Model used in simulations and $B_{r}$ distributions for two coils. . . . . . . . . 52 
3.4 Illustration of the experiments to measure specific conductivity and relative permittivity of a muscle tissue.

3.5 Illustration of the experiments to measure coil's sensitivity. . . . . . . . . 55

3.6 Experimental setup to detect the signal with coil. . . . . . . . . . . . 57

3.7 The signal from the pickup coil in time domain. The signal was expected at 35 and $41 \mu s$ (a), corresponding to the interfaces indicated by the bursts in pulse-echo signal (b). . . . . . . . . . . . . . . . . . . 58

3.8 Sample used for studying the voltage signal f . . . . . . . . . . . . . . 59

3.9 Illustration of the signal detection with electrodes. . . . . . . . . . . . . 60

3.10 Verification of the fact that the electrode signal comes from the pork muscle

3.11 Experiments on the pork sample to verify the effect of the polarity and the electrode signal in absence of the external voltage. . . . . . . . . . . .

3.12 An illustration of the experimental setup to study the effect of the frequency of the applied AC voltage on the electrode signal.

3.13 Experiments to illustrate the behavior of the electrode signal with frequency. Arrows mark the signal from the front and the rear interface between the oil

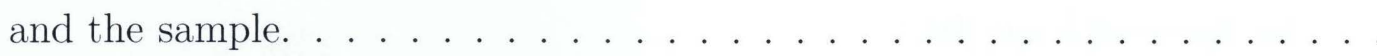

4.1 Heterogeneous sample used in our experiments and the position of the transducer.

4.2 Electrode (a) and acoustic (b) signals from the sample shown on Fig. 4.1. . . 70

4.3 Electrode (a) and acoustic (b) signals from the sample analogous to that shown on Fig. 4.1. 
4.4 (a) Illustration of the experiments with the $10 \mathrm{MHz}$ transducer. (b) Dimensions of the sample in front view. (c) Dimensions of the sample in 3D view .

4.5 Results of the experiments with the $10 \mathrm{MHz}$ transducer. Each row corresponds to a particular flight time between the transducer and the sample. Left column contains are the uvp signals, at right column are the pulse-echo signals. . . . 74

4.6 Spectrum of the electrode signal on Fig. 4.5a. The filtered part is marked with a dashed rectangle. . . . . . . . . . . . . . . . 76 


\section{List of Tables}

2.1 Parameters to calculate the acoustic pressure of the $2.25 \mathrm{MHz}$ transducer at the focal zone. . . . . . . . . . . . . . . . . . . . . . . 32

3.1 Measured values of $\epsilon_{r}$ and $\sigma$ for a slub of pork muscle tissue at several frequencies. 54

A.1 Main acoustic and electromagnetic parameters of the media and materials used in experiments $[2,3,4,5] \ldots \ldots \ldots \ldots \ldots$ 


\section{Chapter 1}

\section{Introduction}

\subsection{Basics of bioimpedance}

\subsubsection{Electrical properties of tissues and their relation to different pathologies}

The bioimpedance of tissues and organs contains rich information on their physiology and pathology. That is why a number of imaging techniques aiming to reconstruct the impedance of biological tissues are under current investigation and improvement[6]. The bioimpedance mapping possesses special ability to distinguish blood, air, body liquid and different composition of tissue [7]. The changes in bioelectrical impedance can be caused by flowing and distribution of blood, exchange of blood and air in the lung, change and movement of body liquid and so on. The bioimpedance technology has the potential to conduct functional evaluations, such as to the estimate function of brain, heart and lung, to study hemodynamics and hemorheology in vivo, to discover cancer in early stage, and to analyze the composition 
of human body [7].

The bioimpedance technology has the advantages of being noninvasive, inexpensive, safe, and providing rich functional information and it is easy to use. Doctors and patients are easy to accept it [7].

The most significant application areas of imaging the electrical impedance today are monitoring lung function [6] with Electrical Impedance Tomography (EIT), mapping the brain activity with Magnetic Impedance Tomography (MIT) [8] and skin and breast cancer detection in EIT [9]. There are several EIT systems avaliable on the market, including Sheffield Mark 3.5 system for breast imaging and GOE MF II system to monitor the respiratory function. No MIT system has reach so far routine use in medicine, although several research groups are working on it. [10]. Another recently developed imaging modality, Magnetic Resonance Electrical Impedance Tomography (MREIT) will be discussed later. The applications of the bioimpedance techniques to cancer imaging contribute a lot to the research interest in this area $[6,11]$. To understand what makes cancer detectable with bioimpedance techniques, the composition of biological tissues in general and specific features of cancerous tissues need to be reviewed, in relation to their macroscopic electrical properties.

\subsubsection{Composition of biological tissues}

A biological tissue is essentially biological cells surrounded by an extracellular fluid [1]. The metabolism between blood capillaries and a tissue is schematically shown in Fig. 1.1. Along with atoms and compound molecules crucial for functioning, blood transports different ions such as $\mathrm{Ca}^{2+}, \mathrm{Mg}^{+}, \mathrm{K}^{+}, \mathrm{Cl}^{-}$. There is a continuous ion exchange between the cells and the 
extracellular fluid, which results in slightly different ion content in extra- and intracellular fluid.

Due to such composition, tissue fluids are considered electrolytes, where the conductivity is due to the ions free to migrate. A conductivitry is a measure of a materal's ability to conduct an electric current. If a potential difference is applied to a conductive material, it will induce a current density $\mathbf{J}$. One can introduce the conductivity $\sigma$ by the following equation:

$$
\mathbf{J}=\sigma \mathbf{E}
$$

Conductivty is the reciprocal of the electrical resistivity $\rho$ and has the SI units of $S \cdot m^{-1}$. The ionic conductivity of an electrolyte solution depends on the charge, mobility and its ionic strength [1]. The conductivity also depends on the temperature, viscosity of the solvent and frequency of the applied electric field, which, for biological tissues, can be described by Debye formula $1.2[1]$ :

$$
\sigma^{*}=\sigma_{\infty}+\frac{\sigma_{0}-\sigma_{\infty}}{1-i \omega \tau}
$$

where $\sigma_{0}$ and $\sigma_{\infty}$ are the electrical conductivities respectively far below and far above the permittivity dispersion region (typically, between roughly below $1 \mathrm{kHz}$ to microvave range [12]), $\omega$ is the angular frequency, $\tau$ is the relaxation time constant. In the Eq. 1.2, the complexity means the disperion with frequency.

Electrical permittivity is a measure of a material's ability to store the charge. Due to their structure, tissues have such ability, along with the ability to conduct the current. The 


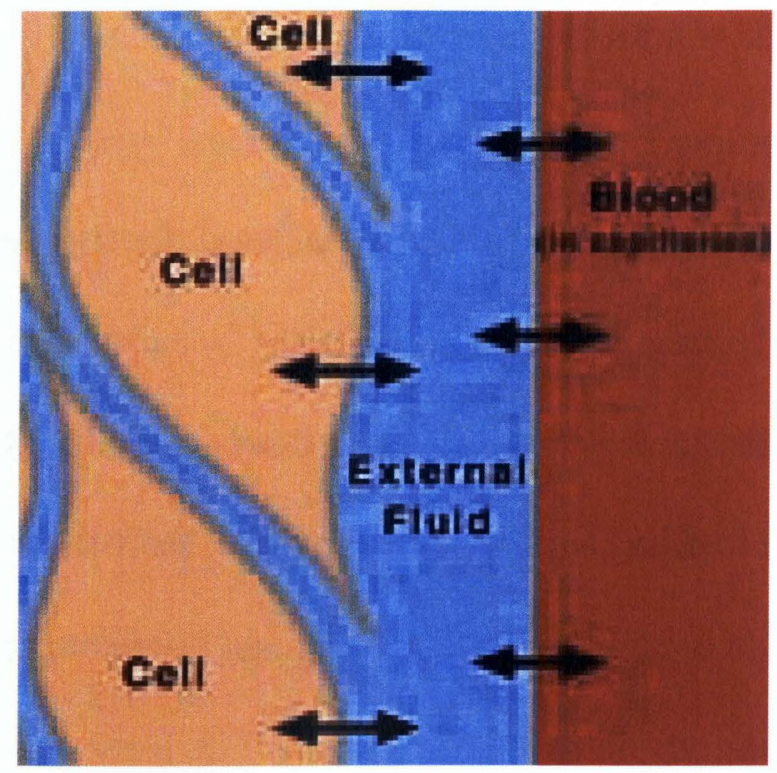

Figure 1.1: Illustration of the metabolism between the blood and the biological tissues.

concept of the relative permitivity was first introduced by Faraday in the 1830s [13] as a factor by which tha capacitance of an air capacitor increases upon introducing a material into it. An equation analogous to Eq. 1.2 can be applied to the complex relative permittivity $\epsilon^{*}$ of biological tissues (Eq. 1.3):

$$
\epsilon^{*}=\epsilon_{\infty}+\frac{\epsilon_{0}-\epsilon_{\infty}}{1+i \omega \tau},
$$

A living cell is a complex structure containing organelles performing different functions needed for its survival. A simplified cell anatomy is shown in Fig. 1.2. The key features to discuss the electrical properties of the biological tissues are the electrolyte nature of the intracellular fluid (cytosol) and the presence of the lipid bi-layer membranes surrounding the cell and its organelles. The cytosol is made up of water, ions and organic molecules and is therefore conductive. The content and structure of each cell depends on its type [12].

The living cell is delimited by the plasma membrane, which provides rigidity and protec- 


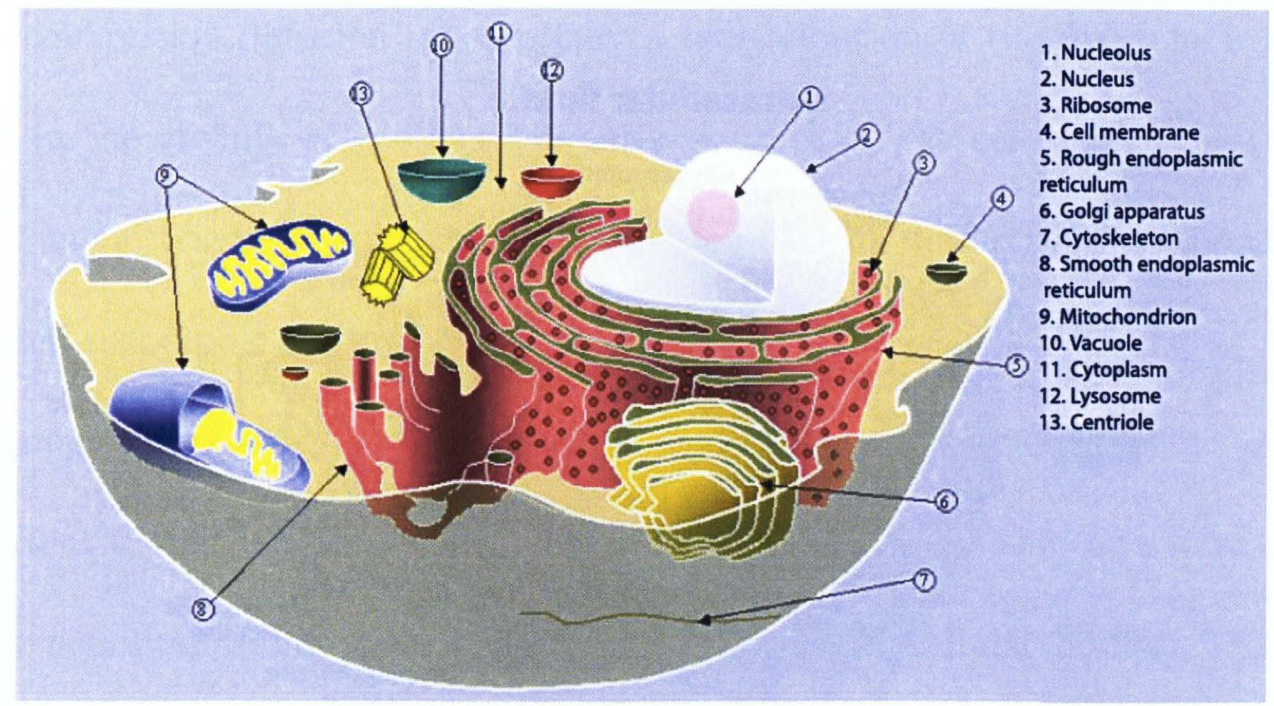

Figure 1.2: Composition of an animal cell.

tion. The cell membrane also binds different cells together. The membrane consists of an asymetric molecular lipid bi-layer which is basically impermeable to ions. However, protein molecules inserted in the bi-layer form channels to transport materials, including ions, across the membrane [12].

The bi-layer membranes are formed by the two layers of lipid molecules. Each molecule has a hydrophilic side (head) and hydrophobic side (tail). The hydrophobic force between the tails makes them attract each other, and the heads end up facing the cytosol and the extracellular fluid. The cell membrane with the transmembrane proteins is shown in Fig. 1.3.

Cell membranes are generally structures with a thickness of about $5 \mathrm{~nm}$. A cell membrane can be modeled as a dielectric slab with the effective capacitance per unit area $\left(C_{m}\right)[14]$

$$
C_{m}=\epsilon_{0} \epsilon_{m} / d,
$$




\section{Extracellular fluid}

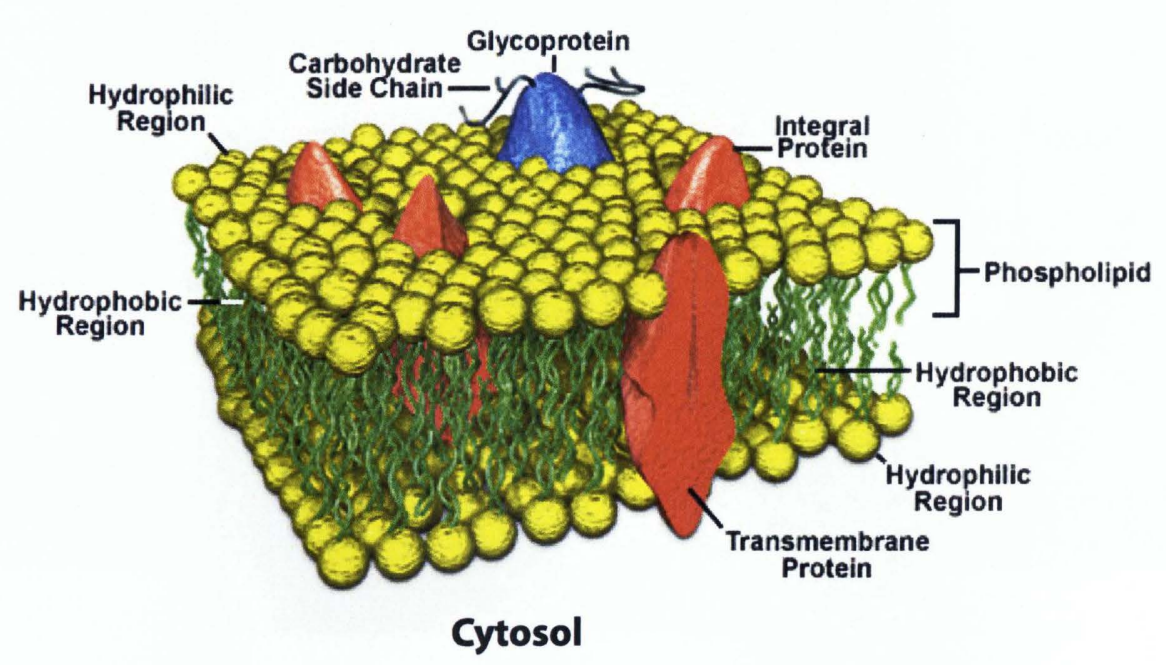

Figure 1.3: Composition of a lipid bi-layer membrane.

where $d$ is the thickness of the membrane and $\epsilon_{m}$ is the permittivity of the membrane-forming material. The membrane capacitance is largely determined by the hydrophobic phospholipid core.

\subsubsection{Frequency dispersion of permittivity of biological tissues}

Tissues are dispersive media in terms of dielectric constant $\epsilon$. Many observations show that in the frequency range from a few $\mathrm{Hz}$ to several $\mathrm{GHz}$, the dielectric permittivity of tissues decreases with the frequency, yet does not follow the Debye Eq. 1.3. Instead, it shows several dispersion domains $[13,12]$. Different types of mechanisms are responsible for the observed disperions.

The $\alpha$ - or low frequency dispersion is characterized by very high permittivity values $\left(\epsilon_{\alpha}=10^{7} \ldots 10^{8}\right)$. This dispersion is caused, at least partially, by the counter-ion diffusion 
effects. A counter-ion diffusion is essentially a redistribution of the free ions in a tissue or electrolyte in the vicinity of the charged surfaces, such as the cell membrane [13]. In the presence of an electric field, opposite charges accumulate on diametrically opposed locations to form a cell-size dipole, which can only vary with low frequencies.

$\beta$-dispersion may occur up to the radio-frequency range, depending on the properties of each particular tissue. The basic mechanism of this dispersion is the capacitive charging of the cellular membranes and those of membrane-bound intracellular bodies. The time constant of the related relaxation (and the exact frequency range) depends on the conduction in the tissues and capacitive coupling through the interfaces. It was established that damaging the cell membranes alters the $\beta$-dispersion. Thus, this dispersion gives information on the cell membrane and intracellular content.

$\gamma$-dispersion is related with the polarization of permanent and induced dipoles in the tissue. Permanent dipoles include dipolar molecules (mainly $\mathrm{H}_{2} \mathrm{O}$ ). Induced dipoles are initially neutral molecules whose charge centers got shifted by the applied field. The relaxation time constant for these molecules is small, and this dispersion usually takes place in the microwave frequency domain. Fig.1.4 illustrates idealized spectrum of permittivity and conductivity of a biological tissue.

\subsubsection{Electrical properties of tissues and cells}

Ellappan et al. [15] electrically modeled cell accounting only for the presence of the cellular (outer) and nucleus (inner) membranes having resistance and capacitance, as well as resistive cytosol and nucleoplasm. It has been established that the inductive properties of tissues are 


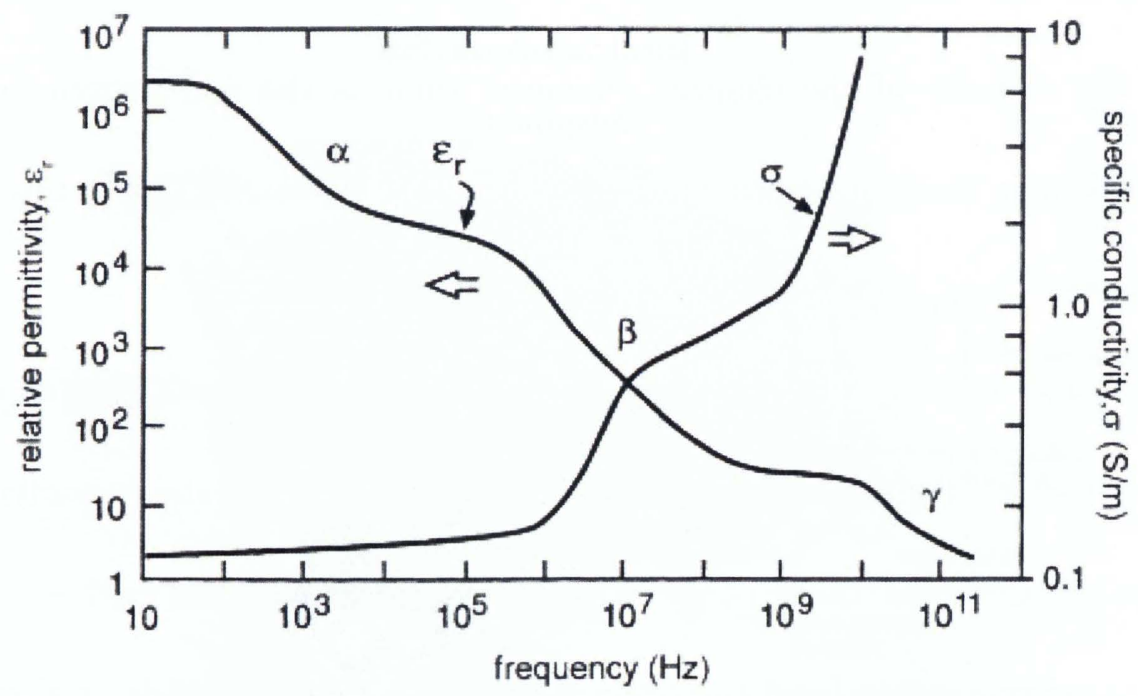

Figure 1.4: Typical frequency dependence of the relative permittivity $\epsilon_{r}$ and conductivity $\sigma$ of a biological tissue [1].

negligible $[6,13]$. The values of the resistors and capacitors are calculated assuming a cubic cell of dimension $10 \mu \mathrm{m}$ and a nucleus of dimension $5 \mu \mathrm{m}$. The resistivities of the cytoplasm and nucleoplasm were assumed to be the same, $100 \Omega \mathrm{cm}$. The specific capacitance of the outer membrane was $1 \mu \mathrm{F} / \mathrm{cm}^{2}$. The capacitance of the nuclear membrane was assumed to be half of that of the outer membrane, because the nucleus membrane consisits of two lipid bi-layers, while the outer membrane is only a single lipid bi-layer.

The circuit depicted on Fig. 1.5 was used to model the response of the outer and nuclear membranes as well as the cell as a whole. The suspension medium is represented by a capacitor and a resistor (left). The nucleus is represented by the membrane capacitance $C_{n 1}$ and $C_{n 2}$ and a resistance $R_{n}$ (right). The cytoplasm is represented by $R_{c 2}$ and the leaky dielectric outer membrane is represented by resistances $R_{c 1}$ and $R_{c 3}$ as well as capacitances $C_{m 1}$ and $C_{m 2}$. The voltage drop across the cell contained two dispersion regions which corresponded 


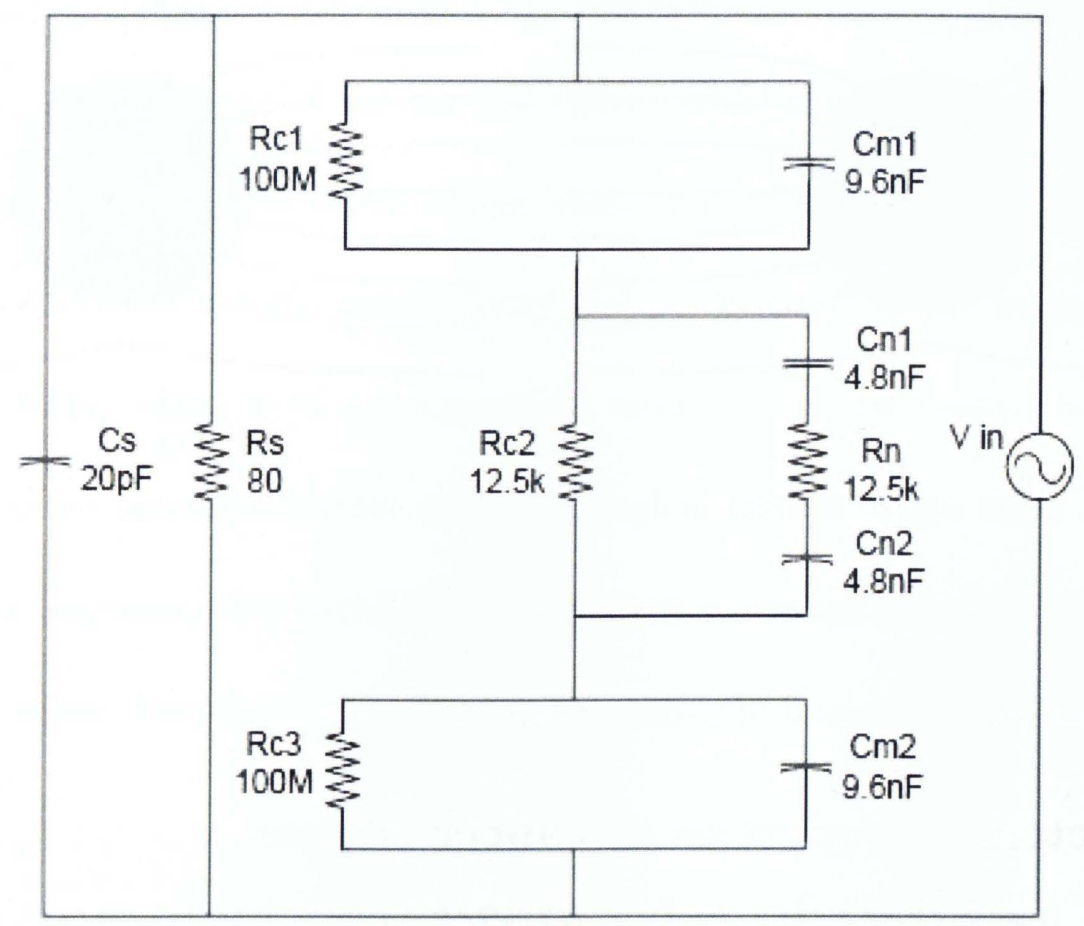

Figure 1.5: Electrical model of a cell with nucleus.

to $\alpha$ - and $\beta$-dispersions. The $\beta$-dispersion occured in the frequency range of $10^{4}-10^{7} \mathrm{~Hz}$. It is caused by the changes in the surface charges and recharging processes occuring in the membrane. Ellappan et al [?] do not distinguish any regions of the $\beta$-dispersion corresponding specifically to the time constant of the cellular and nucleus membranes.

The electric behaviour of the cell membrane results in different propagation of the injected current at low and high frequencies, which is shown on Fig. 1.6. The advantage of the higher frequencies is that the current penetrates the cell interior, and the information from the cellular content can be obtained. 


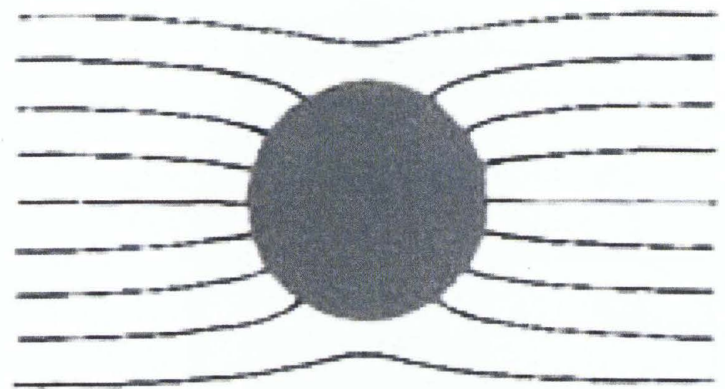

a

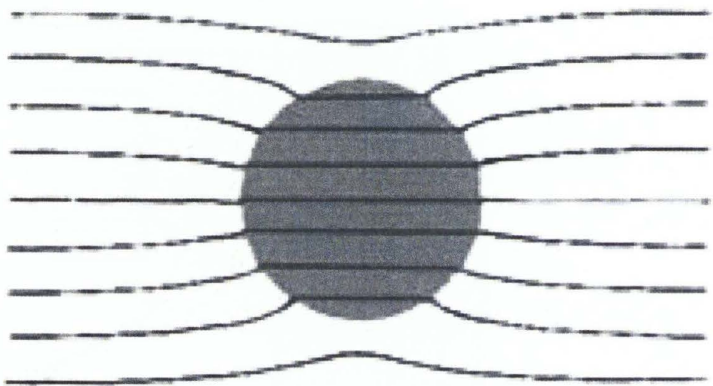

$\mathrm{b}$

Figure 1.6: Distribution of the injected current in the cell at a frequency below (a) and above

(b) the characteristic frequency of $\beta$-dispersion.

\subsubsection{Electrical properties of cancer tissues}

In the most clinically significant frequency range of $\beta$-dispersion, cancerous tissues have electrical properties significantly different from those of normal tissues. This is because the $\beta$-dispersion is mainly affected by the shape of the cells, structure of the cell membranes and the amount of intra and extra cellular water [16]. Electrical impedance within the $\beta$ dispersion range of cancer differs from healty tissues because the cancer cells are different in shape, size and orientation. A statistical study has been done on a number of benign and malignant skin structures [16]. Smith et al [17] measured the conductivity and dielectric permittivity of a VX-2 liver carcinoma and found that the conductivity of this type of cancer is almost an order higher $\left(\propto 10^{-3} \mathrm{~S} / \mathrm{cm}\right)$ than that of a normal liver tissue, while the permittivity is several times lower $\left(10^{4}\right.$ versus $\left.10^{5}\right)$.

A large number of studies has been done on breast cancer tissue. Lazebnik et al [18] did a statistical study on the electrical properties of different breast cancer tissues in a broadband frequency range from 0.5 to $20 \mathrm{GHz}$. Their data was in a good agreement with a model 
based on Cole-Cole interaction. In their study, the effective conductivity of cancerous tissue was 2 orders higher than that of the normal tissue, while the dielectric permittivity of the cancerous breast tissue was an order higher than that of the normal one. Surowiez et al [19] did measurements of specific conductivity and permittivity in the frequency range from $20 \mathrm{kHz}$ to $100 \mathrm{MHz}$, which is of a diagnostical interest. In this study, the difference in a specific conductivity between normal and pathological tissues is one order or higher in the $\mathrm{MHz}$ region. At the same time the dielectric constant is one order higher as well $\left(10^{4}\right.$ versus $10^{3}$ for normal breast tissue).

\section{$1.2 \quad$ Imaging electrical properties of tissues}

\subsubsection{Electrical Impedance Tomography (EIT)}

EIT is a relatively new imaging method that has evolved over the past 20 years. It has the potential to be of clinical value and plays an important role in diagnostics and monitoring of a number of diseased conditions, from breast cancer to lung function. The EIT systems can be divided into single source and multiple source systems. The general structure of single and multiple source systems is schematically shown on Fig. 1.2.1 [6].

The waveform synthesis block produces a sinusoidal waveform. This waveform is then fed to a dual current source, which produces a pair of currents having same magnitudes but opposite polarities. A 2-to- $\mathrm{N}$ multiplexer allows these currents to be applied to one pair of electrodes at a time. Electrode voltages are then measured using multiple differential voltmeters to reduce the range of the signal and fasten up the data acquisition. The voltmeter 


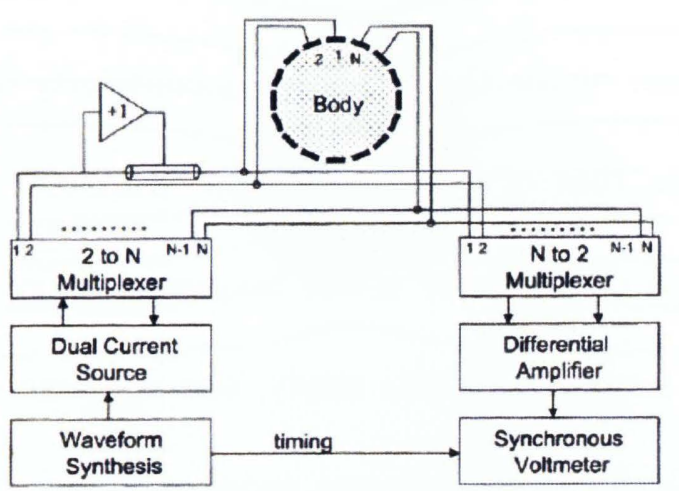

a

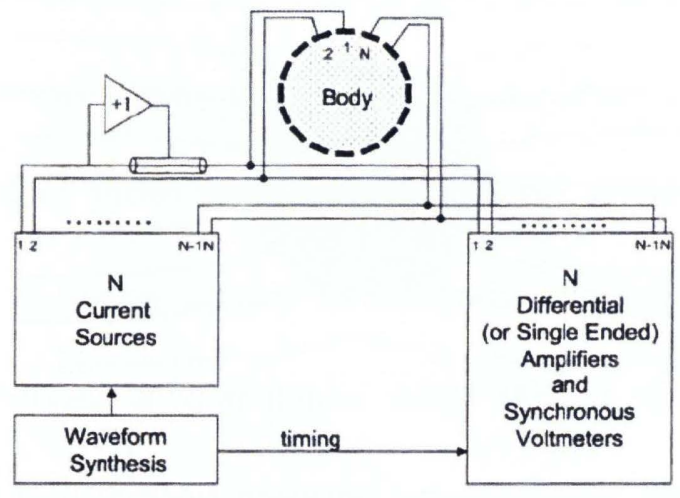

$\mathrm{b}$

Figure 1.7: Single (a) and multiple (b) source EIT systems.

is synchronized with the original waveform in general through a reference waveform from the waveform synthesis block.

The multiple source EIT system (Fig. 1.2.1b) has N current sources - one for each pair of electrodes. Multiple systems operate by applying patterns of currents, that is, each electrode has a current of a particular magnitude running through it. The rest parts of the whole system and the detection principles remain the same.

The forward problem in EIT is formulated based on the quasistatic approach, when the induced magnetic field $\mathbf{H}$ is assumed to be negligible in Maxwell's equations. Then, the Faraday's law becomes

$$
\nabla \times \mathbf{E}=-\frac{\partial \mathbf{B}}{\partial \mathbf{t}}=-\mathbf{i} \omega \mathbf{H}=\mathbf{0}
$$

and therefore

$$
\mathbf{E}=-\nabla \phi .
$$


Next, Ampere's law takes the following form:

$$
\nabla \times \mathbf{H}=\frac{\partial \mathbf{D}}{\partial \mathbf{t}}+\mathbf{J}=\sigma^{*} \mathbf{E}
$$

where $\sigma^{*} \mathbf{E}=(\sigma+\mathbf{i} \omega \epsilon) \mathbf{E}$ includes conduction and displacement current densities. If the divergence is taken on both sides of the Eq. 1.7, we get

$$
\nabla \cdot(\sigma \nabla \phi)=0 \text { in } \Omega
$$

with Dirichlet boundary conditions $\phi=v_{s_{i}}$ under electrode $i$, or Neumann boundary conditions, $\sigma \nabla \phi \cdot n=j_{s_{i}}$ under electrode $i$ and 0 elsewhere. The voltages measured from the $\mathrm{N}$ pairs of electrodes form a vector $V=\left[v_{1}, v_{2}, \ldots, v_{N}\right]$, which can be related to conductivity distribution $\sigma$ by matrix $A_{\sigma}$ :

$$
A_{\sigma} \sigma=V
$$

Because $A_{\sigma}$ is dependent on $\sigma$, the matrix equation 1.9 is nonlinear, with the solution $\sigma$. For the same reason, the inverse problem is also nonlinear. Several linear and non-linear solutions have been developed to reconstruct the impedance. Another problem with EIT is that the solution for the bioimpedance distribution can only be unique if the number of measuring electrodes is infinite; otherwise, a single vector $\mathrm{V}$ can correspond to multiple solutions for $\sigma$.

The examples of EIT systems can be Sheffield system [20] with a single current source which is capable of measuring the current at a range of frequencies from $2 \mathrm{kHz}$ to $1.6 \mathrm{MHz}$. There is also a system to image breast tissue developed by Darmouth group [21]. It has a 
circular electrode array with a frequency range from $10 \mathrm{kHz}$ to $10 \mathrm{MHz}$.

The main application areas of EIT are monitoring lung and brain functions, real-time tracking of the temperature while hyperthermia treatments and detection of breast cancer. At present, EIT does not have the spatial resolution enough to make it acceptable in the field of cancer imaging. However, its key advantage is the temporal resolution, which is in the order of milliseconds [22].

\subsubsection{Magnetic Induction Tomography}

Magnetic induction tomography (MIT) is a noninvasive and contactless imaging modality for reconstructing the conductivity in the target object. In an MIT procedure, series of excitation and receiving coils are placed around an object. Each excitation coil couples an alternating primary magnetic field $\mathbf{B}$ to the object under investigation. This time-varying magnetic field induces eddy currents inside the object. The eddy currents generate a secondary magnetic field, and it becomes $\mathbf{B}+\boldsymbol{\Delta} \mathbf{B}$. The phasor diagram for the primary and the secondary magnetic fields is shown on Fig. 1.8, where the total field $\mathrm{B}+\Delta \mathbf{B}$ lags behind the primary field by an angle $\phi$. If the applied magnetic field has the frequency $\omega$, then it can be shown that the following holds true:

$$
\frac{\Delta \mathbf{B}}{\mathbf{B}}=P \omega \mu_{0}\left(\omega \epsilon_{0} \epsilon_{r}-j \sigma\right)+Q\left(\mu_{r}-1\right),
$$

where $\epsilon_{r}$ is the relative permittivity of the material, $\epsilon_{0}$ is the permittivity of free space and $P$ and $Q$ are geometrical constants.

For biological tissues, $\Delta \mathbf{B}$ is much smaller than $\mathbf{B}$ in magnitude and the conductivity 


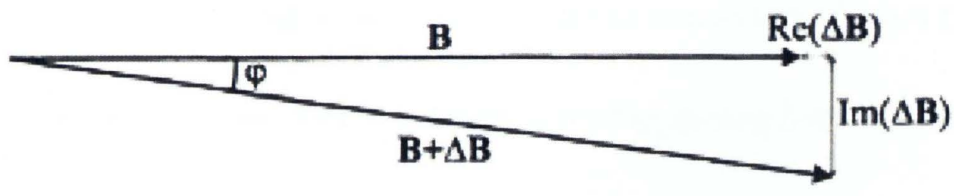

Figure 1.8: Phasor diagram representing the primary $(\mathbf{B})$ and the secondary $(\Delta \mathbf{B})$ magnetic fields in MIT.

term normally dominates, the phase angle can be written [6]

$$
\phi \approx\left|\frac{\Delta \mathbf{B}}{\mathbf{B}}\right| \propto \omega \sigma .
$$

The suggested medical applications of MIT include imaging of the limbs and brain, the measurement of the body composition, and the monitoring of wound healing. The most promising application would be imaging the brain's conductivity, as magnetic field easily penetrates the skull, while some voltage drops across the skull in EIT.

The spatial resolution of an MIT system will depend on the number of independent combinations of exciting and sensing coils [6]. For an array of $N$ coils, which can either act as excitors or receivers, the number of those combinations is $N(N-1) / 2$. It was calculated in [6] for a system designed by Korjenevsky et al [23] consisting of 16 coils (120 independent measurements), that the maximal resolution of such a system would be $1 / \sqrt{120} \approx 9 \%$ of the array diameter (given that the coils are arranged in a ring). So far, the best achievement in MIT can be the invivo images showing internal anatomical structure by the group of A. Korjenevsky. This is, however, not enough to detect cancer at early stages.

The forward mapping can be formulated as 


$$
y=\Psi\left(\sigma^{*}\right),
$$

the discretized non-linear forward mapping of the conductivity matrix $\sigma^{*}$. The inverse problem

$$
\sigma^{*}=\Psi^{-1}(y)
$$

is ill-posed and underdetermined. A uniqueness of the solution for this problem was proven in [24], given that the frequency of the applied $\mathrm{AC}$ voltage is not a resonant frequency of the receiving coils.

\subsubsection{Magnetic Resonance Electrical Impedance Tomography (MREIT)}

In parallel with EIT, another technique has been developed to image the conductivity, which was called magnetic resonance electrical impedance tomography (MREIT) [25]. In MREIT, magnetic resonance phase images are acquired, and the conductivity is reconstructed from this information. First, the applied currents distribute inside the sample, creating magnetic flux density B through Ampere's law. Thus, this magnetic flux density contains information about the conductivity distribution. For alternating currents, the component of magnetic flux density parallel to the main static field (z-component) can be measured by MRI using spin-echo sequence. The current-generated magnetic flux density introduces a phase shift $\phi$ into the image, give as [26]:

$$
\phi(r)=2 \gamma_{c} T_{c} B_{z}(r),
$$


where $\gamma_{c}$ is the gyromagnetic ratio, $T_{c}$ is the duration of each pulse, and $B_{z}(r)$ is the amplitude of z-component of the current-generated magnetic flux density. Thus, only one component of the magnetic field is used to reconstruct the current density. The forward problem in MREIT is essentially governed by the Poisson equation for the current-induced electric potential $U(r)$ with Neumann boundary conditions:

$$
\sigma \frac{\partial u}{\partial n}=\left\{\begin{array}{cc}
J_{s}, & \text { for positive current electrode } \\
-J_{s}, & \text { for negative current electrode } \\
0, & \text { elsewhere }
\end{array}\right.
$$

as well as the Biot-Savart law to calculate the induced magnetic flux density:

$$
\mathbf{B}(\mathbf{r})=\frac{\mu_{0}}{4 \pi} \int_{\Omega} \mathbf{J}\left(\mathbf{r}^{\prime}\right) \times \frac{\mathrm{r}-\mathbf{r}^{\prime}}{\left|\mathbf{r}-\mathbf{r}^{\prime}\right|^{\mathbf{3}}} \mathbf{d v}^{\prime}
$$

The reverse problem is essentially solving the Biot-Savart law equation in matrix form for the $\mathbf{x}$ - and $\mathbf{y}$-components of the current density $\mathbf{J}$, from z-component of $\mathbf{B}$. This is so-called Bz-algorithm.

Just as MRI, MREIT has the advantage of high spatial resolution $(0.75 \times 0.75 \mathrm{~cm}$, as reported in [25]), while being not as affordable as, say, EIT. The areas of application of MREIT are same as those of EIT. MREIT, however, doesn't have temporal resolution of EIT. 


\subsection{Thesis objectives and outline}

The goal of this research is to develop a method to obtain high-spatial-resolution information about the conductivity and permittivity of biological tissues by combining ultrasound and electromagnetic fields. Chapter 2 contains a journal manuscript on the development of an already reported imaging method, Magneto-Acousto-Electrical Tomography, to obtain signal from a tissue-mimicking sample using the dynamic radiation force induced by ultrasound. In this imaging method, the goal is to reconstruct the conductivity of biological tissues. Chapter 3 is focused on imaging the permittivity of biological tissues. It will be shown that acoustic vibrations generate magnetic moments in a polarized dielectric sample which will depend on the value of the relative permittivity. The experimental setup for detecting the magnetic moment from the sample with a pick-up coil and with attached electrodes will be presented. The origin of the signal obtained from probing electrodes will be discussed. Finally, another contrast mechanism, that occurs in a collodial or ionic sample, will be studied by means of ultrasonic vibration potentials (UVPs) from heterogeneous samples. 


\section{Chapter 2}

\section{Differential frequency}

\section{Magneto-Acousto-Electrical}

\section{Tomography (DF-MAET)}

To provide high-spatial-resolution impedance information, a new approach was developed that is called Magneto-Acousto-Electrical Tomography (MAET). It has the contrast of EIT and the spatial resolution of sonography. Our strategy is first to map the current density distribution $\mathbf{J}_{a b}(\mathbf{r})$ that would exist in a sample if a current/voltage source were to be applied through measurement electrodes $a$ and $b$ (Fig. 2.1). Second, having mapped $\mathbf{J}_{a b}$ one can calculate the electrical impedance of the sample. To map the distribution of $\mathbf{J}_{a b}(\mathbf{r})$ experimentally, without directly applying a current/voltage source, we placed the sample in a static magnetic field and focused an ultrasonic pulse on the sample to simulate a point-like current dipole source at the point $\mathbf{r}$ (Fig. 2.2). The vibration of ions in the sample caused by 


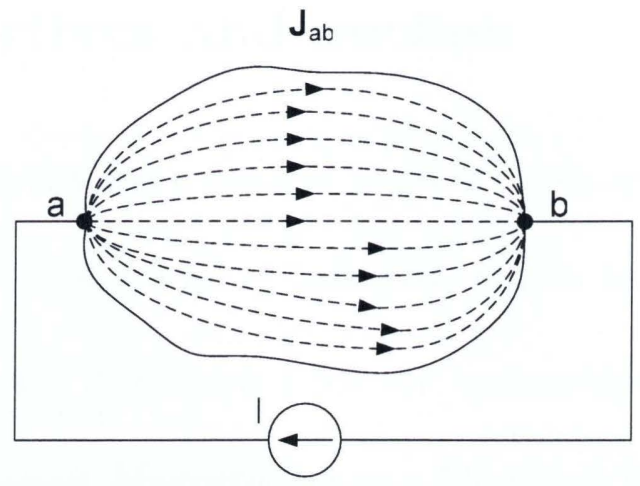

Figure 2.1: Illustration of lead field current density distribution in a sample.

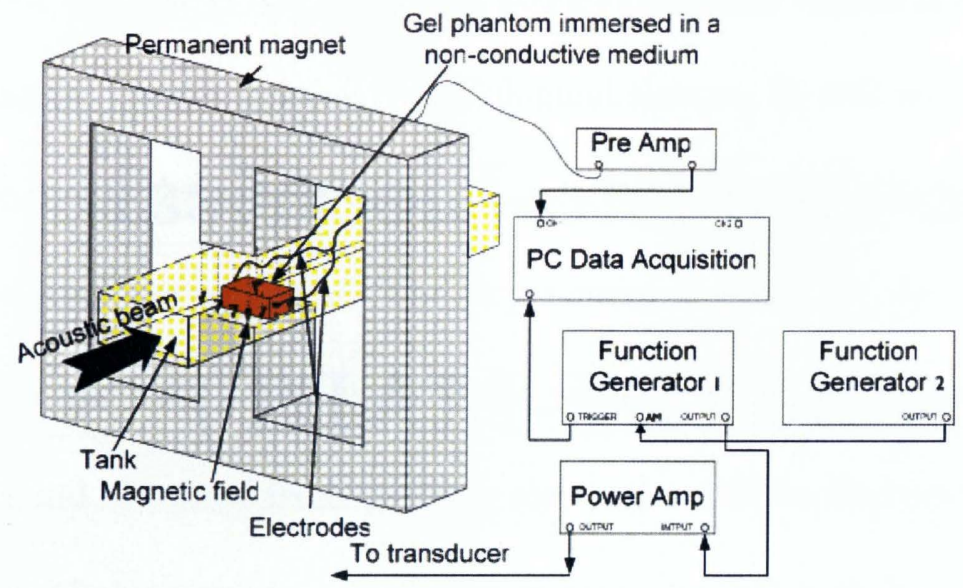

Figure 2.2: MAET experimental setup.

the ultrasound can induce an electric field via the Lorentz-force mechanism. Consequently, a voltage/current signal can be determined via the measurement electrodes [27, 28, 29, 30]. Based on the reciprocity theorem of electromagnetic waves [31], it has been determined determined that the measured voltage/current is proportional to a component of $\mathbf{J}_{a b}$ :

$$
U_{a b}=\int_{V}\left[\left(\mathbf{v} \times \mathbf{B}_{0}\right) \cdot \mathbf{J}_{a b}\right] d^{3} \mathbf{r}^{\prime}
$$


where $U_{a b}$ is the voltage signal, $\mathbf{v}$ is the particle vibration velocity in an ultrasound wave, $\mathbf{B}_{0}$ is the static uniform magnetic field, and $d^{3} \mathbf{r}^{\prime}$ is the volume element inside the sample. Proportionality of the voltage signal to velocity field means that the voltage signal has the same spectrum as the applied ultrasound beam, since $\mathbf{B}_{\mathbf{0}}$ and $\mathbf{J}_{\mathrm{ab}}$ are independent of time. Also, as the velocity is not zero only in the focal spot, the lateral resolution in MAET is limited only by the diameter of the acoustic beam at the focal spot. After scanning the ultrasound beam zone throughout the sample, one can map $\mathbf{J}_{a b}(\mathbf{r})$. The electrical impedance of the sample may be reconstructed from $\mathbf{J}_{a b}(\mathbf{r})$, as was reported previously [32].

We discussed linear MAET experimental results on gelatin samples in [32]. The signal distribution obtained with MAET in those experiments was in good agreement with the results from the numerical simulation for a chosen sample shape. However, in the initial experiments we observed MAET signals only when the ultrasound pulse crossed the interfaces between media with different conductivities. This is because the MAET signal is an integration of $\left(\mathbf{v} \times \mathbf{B}_{\mathbf{0}}\right) \cdot \mathbf{J}_{\mathbf{a b}}$ over the spatial extent of the ultrasound pulse, as shown in Eq. 2.1, and because $\mathbf{v}$ is spatially oscillatory with the period of the ultrasound wavelength, which was $0.67 \mathrm{~mm}$ in our experiments. On one hand, this oscillatory nature of the ultrasonic wave makes the integral value in Eq. 2.1 very small when $\mathbf{B}_{\mathbf{0}}$ and $J_{a b}(\mathbf{r})$ are approximately constant within the spatial extent of the short ultrasound pulse. This renders the signal inside the uniform sample undetectable with our current setup. On the other hand, at the interfaces between media having different conductivities, there is a step change in $\mathbf{J}_{\mathbf{a b}}$. This yields an integral value of $\left(\mathbf{v} \times \mathbf{B}_{\mathbf{0}}\right) \cdot \mathbf{J}_{\mathbf{a b}}$ which is much larger at the interfaces than in the uniform interior of the sample.

Fig. 2.3 illustrates cancellation of the signal from the homogeneous interior and its 
presence only at the interfaces [1]. The conductive gelatine sample is shown in Fig. 2.3a. It has a centered rectangular cavity which was filled with oil when the sample was immersed in oil. The transducer axis is along the z-axis and the scanning is along the x-axis. When the ultrasound beam is focused at point A, it does not intersect with the cavity and crosses only two interfaces. The voltage signal when the ultrasound is focused at point A is shown in Fig. 2.3b; it has two pulses at $59 \mu s$ and $79 \mu s$ corresponding respectively to the front and rear faces of the sample when looking from the transducer. When the ultrasound is focused at point B the MAET signal has four pulses, corresponding to the four interfaces, since the ultrasound beam crosses the cavity when the ultrasound is focused at point B. Between the pulses corresponding to the interfaces, there is no obvious MAET signal, which is due to the signal cancellation caused by the oscillatory nature of ultrasound.

As was mentioned in the theory section of [32], we should measure the current density at every point in the sample in order to reconstruct its electrical impedance. Therefore, detection of the signal from the interior is a goal we need to achieve to make MAET a quantitative imaging method.

\subsubsection{DF-MAET signal induced by radiation force}

The radiation pressure occurs in media with nonlinear relation between pressure and density [33]. For a case of sinusoidal wave, a force will be exerted that consisits of two components. The first one oscillates in space and time with an average of zero and creates time varying acoustic pressure acting on the body. The second component, the radiatin pressure, is steady. In a fluid displaced in the direction of the pressure gradient, an acoustic streaming will be 
observed.

The radiation pressure can be understood from the quantization of the acoustic field, that is, representing the field as phonons of energy E. Each phonon carries the momentum of $p=\frac{E}{c}$, where $c$ is the acoustic speed. If it incident on an ideal reflecting plane, it will transfer the momentum of $2 \cdot \frac{E}{c}$. Averaging this change in the momentum over a short period of time $T$, we will obtain the radiation force:

$$
f_{r}=\frac{2<P>_{T}}{c},
$$

where $\langle P\rangle_{T}$ is the transferred power averaged over time $T$. The radiation pressure is simply derived by dividing Eq. 2.2 by the irradiated area.

Similarly, for the ideal absorbing plane, the momentum transferred by each phonon is $\frac{E}{c}$, and

$$
f_{r}=\frac{<P>_{T}}{c}
$$

In case if the medium lets an acoustic wave propagate, the radiation force is proportional to the attenuation coefficient of the medium.

In this Chapter, we report our utilization of the radiation force induced by dual-frequency ultrasound to generate DF-MAET signals from the interior of samples by taking advantage of the unidirectional nature of the radiation force. When an acoustic wave is propagating in a medium, part of its energy is attenuated through absorption and scattering in the medium. Consequently, the momentum carried by the attenuated energy (or phonons) is transferred to the medium, and a radiation force is exerted upon the medium. The radiation force can 
be static (unidirectional), or dynamic (oscillating). In the static case, the radiation force contains a static component pointed in the direction of wave propagation.

In recent years, ultrasound-induced radiation force has been applied to studying the viscoelastic properties of biological tissues using novel imaging methods [34, 35, 36, 37]. For example, in vibro-acoustography (VAG) [35] two overlapping continuous-wave ultrasound beams with slightly different frequencies are used to exert a force in the object at the beat frequency (kHz-range). The acoustic emission resulting from the object's vibration is measured and used to map the mechanical properties of the object. A similar principle is used to monitor tissue temperature [34]. In shear wave elasticity imaging (SWEI) [36], the ultrasound radiation force is used to generate shear waves in tissues, and the speed of the shear wave is monitored to obtain the distribution of Young's modulus. In acoustic radiation force impulse imaging (ARFI), a pulsed radiation force generated by a pulsed ultrasound beam results in displacement within tissues, which is used to probe the mechanical properties of tissues [38].

All the methods described above offer high spatial resolution determined by the size of the focal spot; for example, a study on VAG [39] produces images with a resolution of $1.2 \times 1.2$ $\mathrm{mm}$.

The motivation of these experiments is to check if we can use the radiation force to generate a DF-MAET signal with high spatial resolution. We used amplitude modulation of pressure to generate a dynamic radiation force.

It has been shown that the dynamic radiation force acts on an object located in two superimposed acoustic fields with angular frequencies $\omega_{1}$ and $\omega_{2}$ [35]. This radiation force can be computed as an integral: 

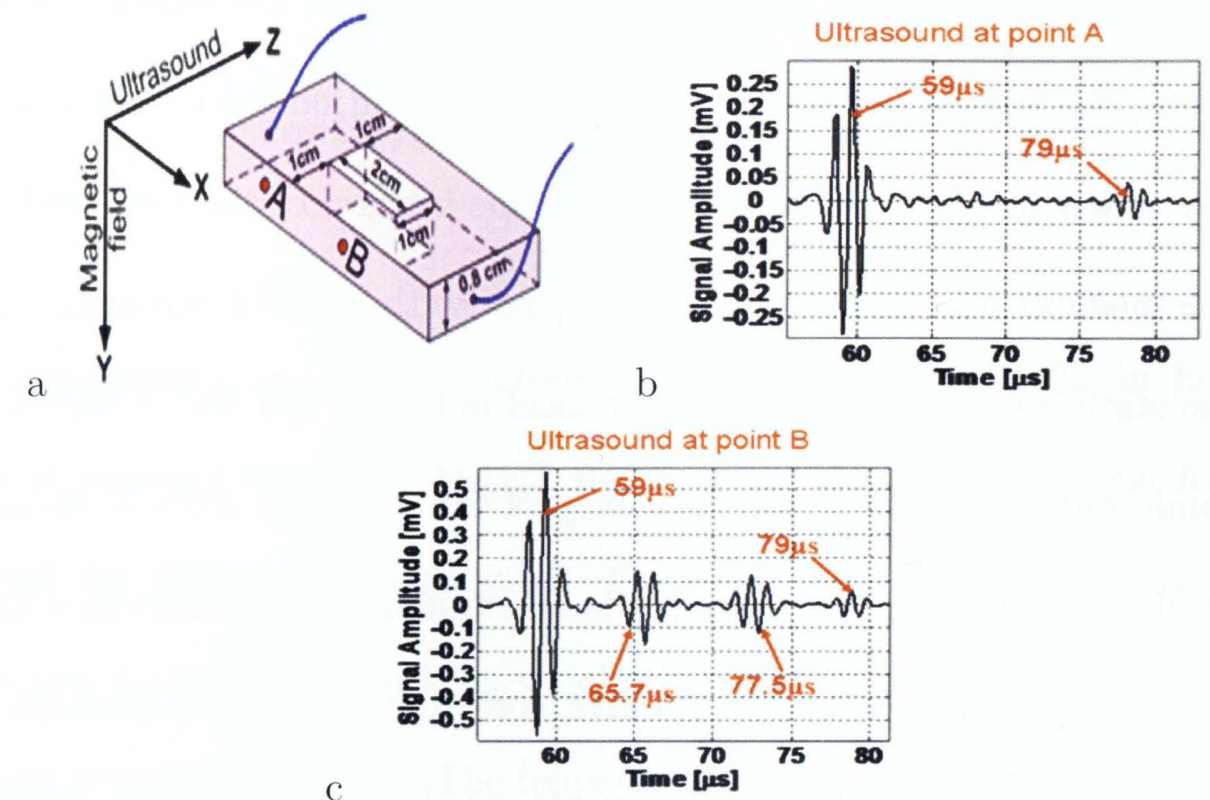

Figure 2.3: An experiment to illustrate the cancellation of the signal from the homogenous interior. A conductive gelatin sample with oil-filled cavity (a), and signals when the beam was focused at the points A (b) and B (c) are shown [1]. 


$$
F_{r}(t)=d_{r} \iint_{S}<\mathrm{E}_{\text {focal }}>_{T} d x d y=C d_{r} \cos \delta \omega t
$$

where $S$ is a projected area, $\left\langle\mathrm{E}_{\text {focal }}\right\rangle_{T}$ is the energy density averaged over a short time $\mathrm{T}, d_{r}$ is a coefficient related to the reflection and scattering properties of the object, $C$ is a constant, and $\delta \omega=\left|\omega_{1}-\omega_{2}\right|$ is the angular difference frequency.

Unlike the incident ultrasonic wave that oscillates in space frequently (for a $2.25 \mathrm{MHz}$ ultrasound wave the spatial period is $0.67 \mathrm{~mm}$ ), the radiation force does not oscillate or oscillates very slowly in space. Therefore, the volume integral of the force and the induced motion in the medium will not be zero. Consequently, the MAET signal in Eq. 2.1, generated by the static radiation force will not cancel out. We can find a similar conclusion for the dynamic radiation force if the frequency of the radiation force is chosen properly. For example, if the dimension of the sample is $10 \mathrm{~cm}$ along the transducer's axis, and the radiation force has a frequency of below $7.5 \mathrm{kHz}$, corresponding to a wavelength larger than $20 \mathrm{~cm}$ in soft tissue, the phase change across the whole 10-cm sample is smaller than $\pi$. Consequently, the integration of the MAET signals from different points of the sample will not cancel out.

In the current study, we used the amplitude modulation of pressure to generate the dynamic radiation force to induce the DF-MAET signals with a good signal-to-noise ratio $(\mathrm{SNR})$ :

$$
p=p_{0} \cos 2 \pi f_{m} t \cdot \cos 2 \pi f_{0} t
$$

where $p_{0}$ is the pressure amplitude, $f_{m}$ is the modulation and $f_{0}$ is the carrier frequency. 
Following from the basic trigonometry, we have

$$
p=\frac{p_{0}}{2}\left(\cos \left[2 \pi\left(f_{0}-f_{m}\right) t\right]+\cos \left[2 \pi\left(f_{0}+f_{m}\right) t\right]\right) .
$$

Now, as the radiation force is proportional to the $\left\langle E_{\text {focal }}\right\rangle_{T}$ in Eq. 2.4, we can further expand the square of the sum in Eq. 2.6, and then rewrite the product of cosines as:

$$
\begin{array}{r}
\cos \left[2 \pi\left(f_{0}-f_{m}\right) t\right] \cos \left[2 \pi\left(f_{0}+f_{m}\right) t\right]= \\
\frac{1}{2}\left(\cos 4 \pi f_{m} t+\cos 4 \pi f_{0} t\right)
\end{array}
$$

In our experiments we are only interested in the term with $4 \pi f_{m}$ in Eq. 2.7. This component of pressure induces a MAET signal at the double modulation frequency $2 f_{m}$, which we call the difference frequency $\delta f$. Therefore, according to Eq. 2.1, if $f_{0}$ is in the MHz range and $f_{m}$ is about $1-10 \mathrm{kHz}$, we obtain a voltage signal with frequency $2 f_{m}$ from the electrodes $a$ and $b$ in Fig. 2.2. The frequency of the radiation force in DF-MAET is low enough to avoid the cancellation of the MAET signal that occurs in the linear MAET in a homogeneous sample.

In addition, we investigated the contribution of other possible sources of the DF-MAET signal. They are the nonlinear effects in piezo ceramics [40] and the parametric effect in a medium [33]. These effects are briefly described below.

It is known that the relationship between polarization and the applied electric field in piezoelectrics is nonlinear [40], i.e., there is a hysteresis loop between the polarization and the applied electric field. In this loop, the polarization depends on both the magnitude of the applied electric field and its square. Therefore, when the transducer is driven by the electric 
signal with two frequencies $\omega_{1}$ and $\omega_{2}$, there will be an acoustic wave at the differential frequency (DF) emitted by the transducer, due to the quadratic term in the polarization. This wave will propagate from the transducer to the sample and can induce the DF-MAET signal.

The parametric effect is another mechanism of generating a nonlinear acoustic wave [41]. As shown in [33], there is a non-zero frequency difference pressure when fields with frequencies $\omega_{0 a}$ and $\omega_{0 b}$ are applied together and overlap. In the case of a planar wave, this nonlinear pressure can be expressed as

$$
p_{-}(x, t)=-\frac{\beta p_{0 a} p_{0 b} \omega_{-} x}{2 \rho_{0} c_{0}{ }^{3}} \sin \omega_{-} t
$$

where $\beta$ is a medium-specific coefficient of nonlinearity, $p_{0 a}$ and $p_{0 b}$ are the amplitudes of the two incident waves, $\omega_{-}=\left|\omega_{0 a}-\omega_{0 b}\right|$ is the differential angular frequency, $x$ is the distance from the planar transducer to a point in space. Nonlinear waves due to the parametric effect propagate through the sample and can induce the DF-MAET signal. Therefore, we have to consider the parametric effect as a possible source of the DF-MAET signal.

\subsection{Methods and experimental setup}

Fig. 2.2 shows a diagram of the experimental setup. The gelatin sample is placed between the two poles of the permanent magnet with a static field of $0.2-0.3 \mathrm{~T}$. The function generator 1 is used to produce millisecond sinusoidal bursts with the central frequency of the focused transducer, which is $2.25 \mathrm{MHz}$. The focal length of the transducer is $10.2 \mathrm{~cm}$. The function generator 2 modulates the amplitude of the sinusoidal bursts. These bursts are 
then amplified by the power amplifier and are sent to drive the transducer. The ultrasound beam transmitted by the transducer is incident normally to the faces of the slab samples. The function generator synchronizes the data acquisition card in a PC with the excitation of the transducer. The voltage signal is collected by the electrodes, amplified, and acquired by the data acquisition card. For the coupling of acoustic waves, both the transducer and the sample are immersed in a tank of oil. The transducer scanned along a line or in a plane perpendicular to the transducer axis. The step size of scanning varied in our experiments from 1 to $2 \mathrm{~mm}$.

The shape of the sample for this study was similar to the one in our previous study [32]. As shown in Fig. 2.8, our samples were slabs of gelatin with narrow bridges in the middle with two electrodes $a$ and $b$ were placed on both sides. We scan the ultrasound beam across the central bridge part of the sample to obtain MAET images.

To extract the DF signal of interest, we took the Fourier Transform of the voltage signal and the magnitudes of the spectrum at the DF $\delta f=2 f_{m}$ were computed for each scanned point. These magnitudes composed a 2D matrix displayed as a DF-MAET image.

\subsection{Characterization of the acoustic field}

We performed measurements of the acoustic pressure from a $2.25 \mathrm{MHz}$ transducer in the focal zone. The hydrophone was placed in the focal spot $10.2 \mathrm{~cm}$ away from the transucer. The area was scanned with a $1 \mathrm{~mm}$ step in $\mathrm{x}$ - and $\mathrm{y}$-direction. The results are shown in Fig. 2.4 .

By using the calibration data for the hydrophone, the peak pressure was estimated to 


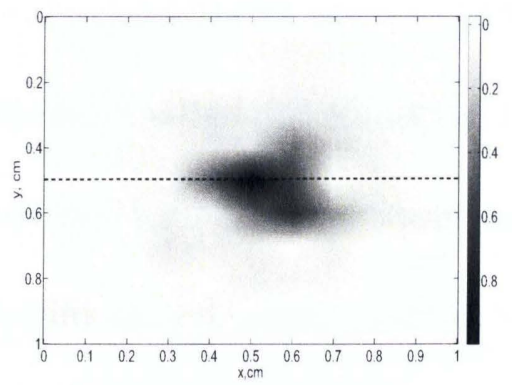

a

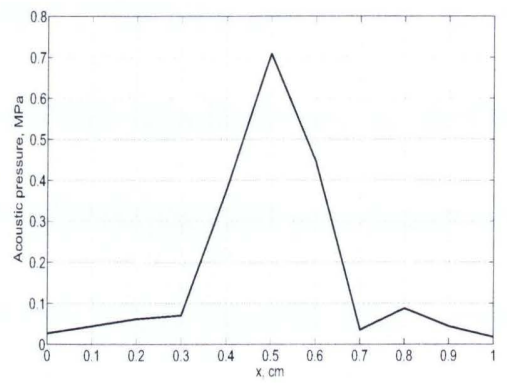

$\mathrm{b}$

Figure 2.4: The 2D image of the pressure distribution of a $2.25 \mathrm{MHz}$ transducer in the focal zone (a) and the line plot across the dashed line (b)

be $0.7 \mathrm{MPa}$ in the focal zone. This value can be also estimated from theory. We used the following equations with the parameters listed in Table 2.1.

$$
\text { Power }=\frac{V_{r m s} \cos \phi}{z_{i n p}}
$$

The power is related to the field intensity in the focal zone as

$$
\text { Power }=I \pi \frac{B D^{2}}{2},
$$

and the intensity is related to the acoustic pressure as

$$
I=\frac{P^{2}}{2 z_{a c}} .
$$

Here, Power is an electric power applied to transducer, $V_{r m s}$ is the root-mean-square voltage, $z_{i n p}$ is the absolute value of the input impedance of the transducer, $\phi$ is the phase of the input impedance, $B D$ is the beam diameter in the focal zone, $I$ is the intensity of the acoustic field, $z_{a c}$ is the acoustic impedance of water, $P$ is the acoustic pressure.

The theoretical estimation for the pressure, $0.074 \mathrm{MPa}$, is 1 order lower than that measured with hydrophone. The reason for that might be the improper hydrophone calibration. 


\begin{tabular}{cccccccc} 
Parameter & $V_{r m s}$ & $\phi$ & $z_{i n p}$ & $\mathrm{BD}$ & $I$ & $z_{a c}$ & $P$ \\
\hline Value & $0.286 \mathrm{~V}$ & 0 & $50 \Omega$ & $2 \mathrm{~mm}$ & $0.18 \mathrm{~W} \cdot \mathrm{cm}^{-2}$ & $1.54 \times 10^{6} \mathrm{~kg} \cdot \mathrm{m}^{-2} \cdot \mathrm{s}^{-1}$ & $0.074 \mathrm{MPa}$
\end{tabular}

Table 2.1: Parameters to calculate the acoustic pressure of the $2.25 \mathrm{MHz}$ transducer at the focal zone.

The $-3 \mathrm{~dB}$ beam diameter was calculated to be $2 \mathrm{~mm}$. The estimations of the intensity of the field for the voltage values actually applied to the transducer are given in the Discussion section of Chapter 2. We did not perform measurements for the power-amplified signal due to the possibility of damaging the hydrophone.

\subsection{DF-MAET experimental results}

In this section, we will present DF-MAET images and show that the radiation force is the main source of the DF-MAET signal. This will be done by investigating the dependance of the signal on both the DF $\delta f=2 f_{m}$ and the density of inclusions introduced into the samples to create a local change in the mechanical properties. Also, we will discuss the spatial resolution in DF-MAET to prove that the radiation force is the source of the DFMAET signal. Finally, we will study the influence of the parametric effect in the medium on the DF-MAET signals. 


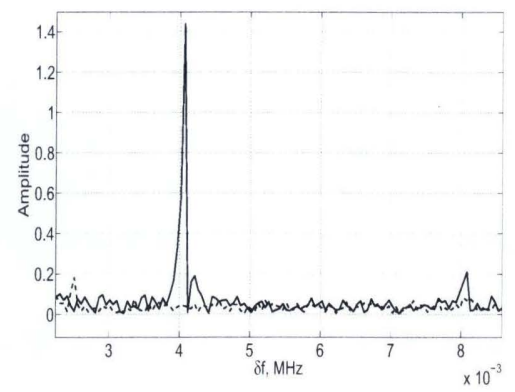

Figure 2.5: $4 \mathrm{kHz}$ component of the signal from the sample (solid line) and empty space (dashed line).

\subsubsection{Dependence of the amplitude of the DF-MAET signal on the modulation frequency}

To study the frequency dependence of the DF-MAET signal, we applied a 15-ms tuned burst of a $2.25 \mathrm{MHz}$ sinusoidal wave modulated at $0.18-10 \mathrm{kHz}$ to drive the transducer. The peak power of the bursts was $20 \mathrm{~W}$. After two-stage 20,000-times amplification, the MAET signal was acquired by the Gage card and averaged 20 times. The temporal spectrum of the MAET signal when ultrasound is focused at the gelatin sample for an acquisition with a modulation frequency of $2 \mathrm{kHz}$ is shown in Fig. 2.5 by the solid line. To verify that the $4-\mathrm{kHz}$ peak is related to the sample, we also show the spectrum (dashed line in Fig. 2.5) of the signal from the detection electrodes when the ultrasound is focused at a point in the nonconductive medium, away from sample. Comparison of the two lines shows that the $4-\mathrm{kHz}$ signal is generated in the conductive sample.

The dependence of the DF-MAET signal on the difference frequency was also studied. The measured DF-MAET signals are shown in Fig. 2.6a and Fig. 2.7 (squared). As can be seen from Fig. 2.6a, the DF-MAET signal decreases nonlinearly up to the modulation 


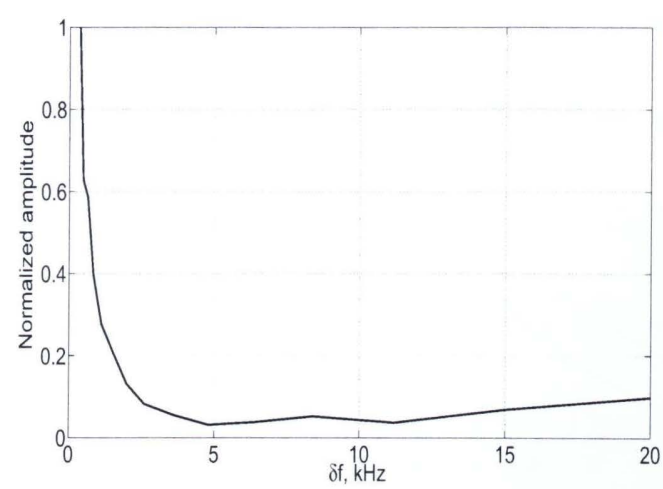

a

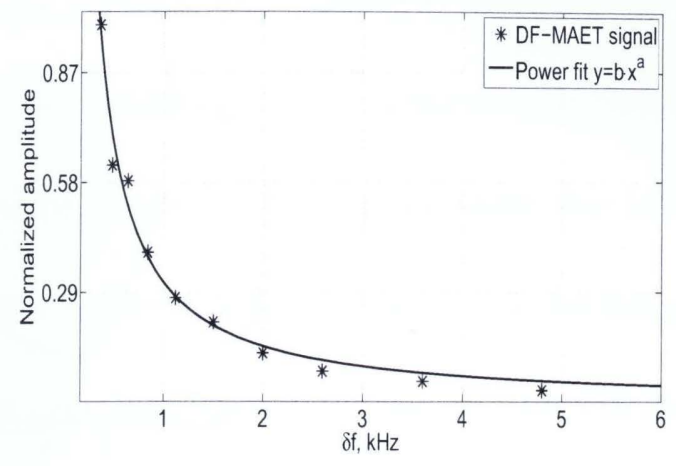

b

Figure 2.6: Spectrum of DF-MAET signal (a) and its fit with the power function $y=b x^{a}(b)$ frequency of $3.2 \mathrm{kHz}$ and increases after that. This can be seen more clearly in Fig. 2.7. The decrease of the signal can be explained by studying the vibration speed of the sample caused by the radiation force. Since we used a $2 \%$ gel and assumed that the shear modulus and viscosity are negligible, Newton's second law for a unit volume sample vibrating at a DF $\delta f$ can be expressed as:

$$
F_{r}(t)=\rho \frac{d}{d t}\left(v_{0} e^{j 2 \pi \delta f t}\right)=\rho v_{0} j 2 \pi \delta f e^{j 2 \pi \delta f t},
$$

where $v_{0}$ is the velocity of vibrations due to the radiation force, $\rho$ is the density of the sample, and $F_{r}(t)$ is the radiation force on the volume. From Eq. 2.1, we have $U_{a b} \propto v_{0}$, which can be combined with Eq. 2.12 to give

$$
U_{a b} \propto \frac{1}{\rho \delta f}
$$

Thus, the important properties of the DF-MAET signal caused by the radiation force are: it is proportional to $\frac{1}{\delta f}$, which will be verified by data-fitting of spectroscopic measurements shown in Fig. 2.6a, and it is also proportional to $\frac{1}{\rho}$. 


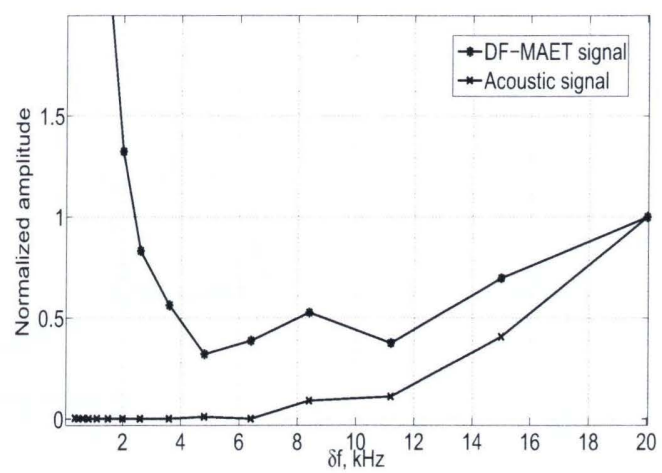

Figure 2.7: Normalized amplitudes of hydrophone acoustic and DF-MAET signals plotted against modulation frequency to show the correlation between them when $\delta f$ is larger than $6.4 \mathrm{kHz}$.

Next, the power-fit $y=b x^{a}$ in MATLAB was applied to the DF-MAET signal in Fig. 2.6a. We obtained a=-0.91 with R-square of 0.97 (a should be equal to -1 from the theoretical prediction). The fitting curve and the experimental data are shown in Fig. 2.6b.

However, the radiation force model fails to explain the increase of the DF-MAET signal with $\delta f$ when $\delta f$ is larger than $6.4 \mathrm{kHz}$, because the DF-MAET signal is predicted to decrease with $\delta f$ monotonically. This suggests that a mechanism other than the radiation force dominates the generation of DF-MAET signals after $6.4 \mathrm{kHz}$. To explain the increase of DF-MAET signal with $\delta f$ when $\delta f$ is larger than $6.4 \mathrm{kHz}$, an acoustic receiver was placed behind the sample to measure the difference frequency acoustic waves. For comparison, we plot the dependance on frequency of both the hydrophone and the DF-MAET signals in Fig. 2.7. Both the DF-MAET signal and the hydrophone signal rise after $6.4 \mathrm{kHz}$. This suggests that the DF-MAET and hydrophone signals are correlated after $\delta f=6.4 \mathrm{kHz}$. The possible mechanisms other than the radiation force to generate the acoustic waves and DF- 
MAET signals at frequencies higher than $6.4 \mathrm{kHz}$ include the nonlinear acoustic emission from the transducer and the parametric effect in the medium. The nonlinear increase of the acoustic signal with frequency shows that its source is the crystal rather than the medium, since the nonlinear pressure arising in the medium is directly proportional to the frequency difference, according to Eq. 2.8. Therefore we conclude that the rise in the DF-MAET signal after $\delta f=6.4 \mathrm{kHz}$ is due to the nonlinear acoustic emission of the transducer caused by the nonlinear piezoelectric response in the piezo crystal. This will further be confirmed by analyzing the resolution at different frequencies in the following section.

In summary, the measured signal for $f_{m}$ less than $3.2 \mathrm{kHz}$, when the acoustic emission is not noticeable, agrees approximately with the prediction of the radiation force model. When $f_{m}$ is larger than $3.2 \mathrm{kHz}$, the DF acoustic emission from the transducer dominates in the DF-MAET signal. As will be shown in the following section, the two different sources of the DF-MAET signal will give different spatial resolutions, and the radiation force is more desirable due to its high spatial resolution. DF acoustic emission from the piezoelectric crystal can be eliminated by using a dual-element transducer rather than a single-element one as used in our present experiments. This will be addressed in details in the Discussion section.

\subsubsection{Spatial resolution in DF-MAET}

To study the spatial resolution in the DF-MAET we scanned the 8-mm high bridge along the vertical line in Fig. 2.8 from point A to point B. The location of the starting point A was selected after a vertical scan of the whole bridge, as the point of maximum signal. 


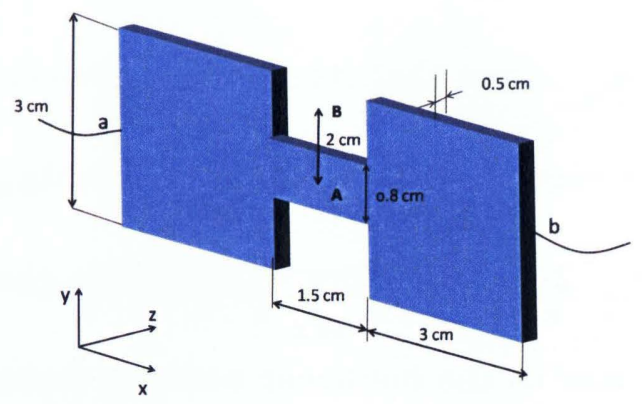

Figure 2.8: Diagram of the phantom to study the resolution in the DF-MAET.

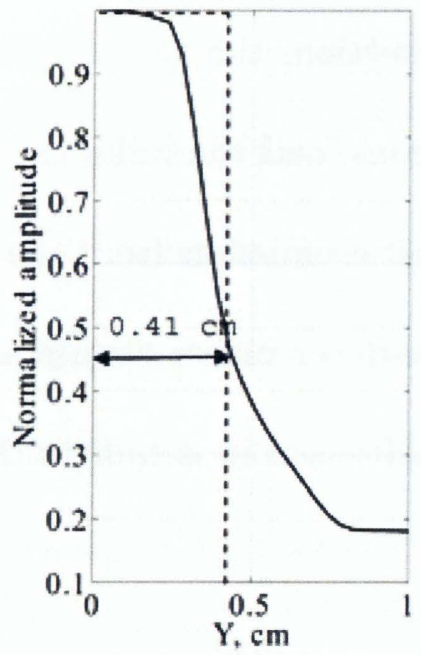

a

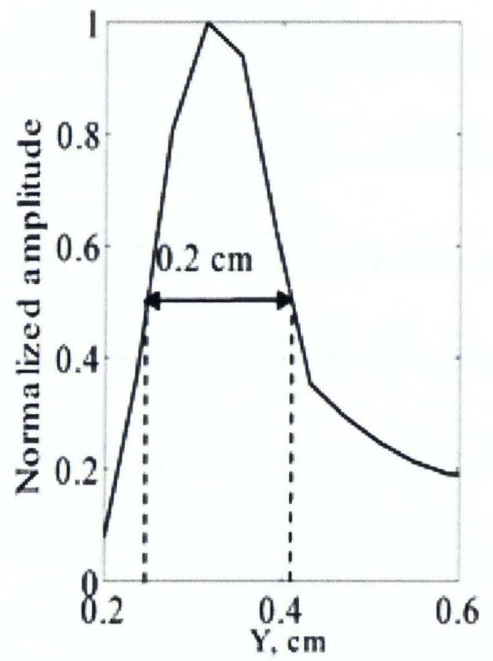

$\mathrm{b}$

Figure 2.9: Edge-spread (a) and line-spread functions (b) of the DF-MAET imaging system for $f_{m}=1 \mathrm{kHz}$. 


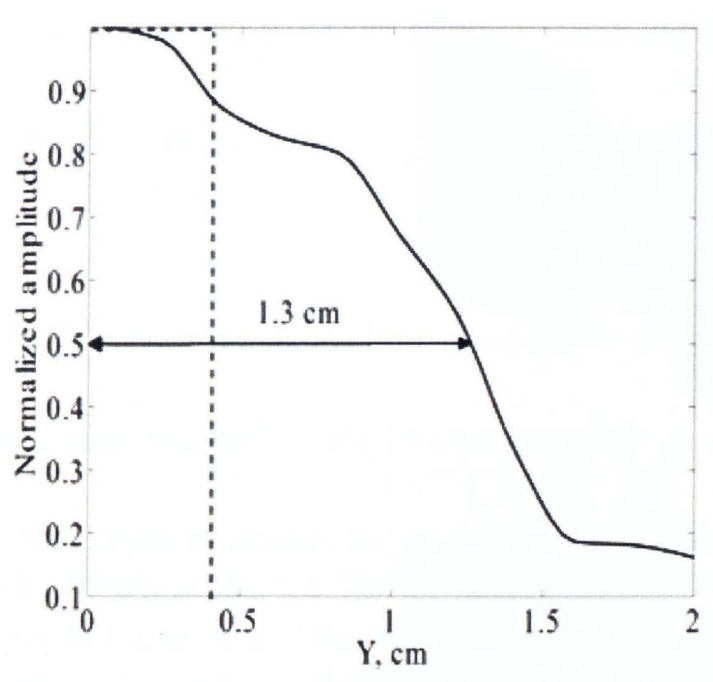

a

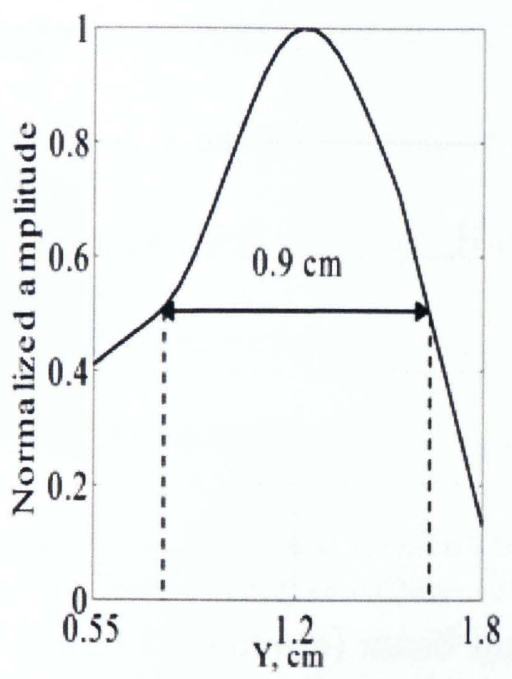

$\mathrm{b}$

Figure 2.10: Edge-spread (a) and line-spread functions (b) of the differential MAET imaging system for $f_{m}=43 \mathrm{kHz}$.

We used a modulation frequency $f_{m}$ of $1 \mathrm{kHz}$ and $43 \mathrm{kHz}$ and a carrier frequency $f_{0}$ of 2.25 MHz. The line plots of the original MAET signal represent the edge-spread function of the imaging system. They were smoothed using the cubic spline interpolation function provided by MATLAB. The edge-spread functions (ESF) and their derivatives, the linespread functions (LSF), for modulation frequencies $f_{m}$ of $1 \mathrm{kHz}$ and $43 \mathrm{kHz}$ are shown in Fig. 2.9 and 2.10, respectively.

From the line plot obtained at the difference frequency $2 \mathrm{kHz}\left(f_{m}=1 \mathrm{kHz}\right)$, the halfwidth-half-maximum (HWHM) of ESF was $0.42 \mathrm{~cm}$ (Fig.2.9a). The line plot at the difference frequency $86 \mathrm{kHz}\left(f_{m}=43 \mathrm{kHz}\right.$, Fig. $\left.2.10 \mathrm{~b}\right)$ yields HWHM of ESF of $1.3 \mathrm{~cm}$. Next, the fullwidth-half-maximum (FWHM) of LSF, which characterizes the spatial resolution, was found to be $0.2 \mathrm{~cm}$ for $f_{m}=1 \mathrm{kHz}$ and $0.9 \mathrm{~cm}$ for $f_{m}=43 \mathrm{kHz}$, as shown in Fig. $2.9 \mathrm{~b}$ and $2.10 \mathrm{~b}$, respectively. Thus, at $43 \mathrm{kHz}$ the resolution is much poorer than at $1 \mathrm{kHz}$. Specifically, when 
$f_{m}=1 \mathrm{kHz}$, the resolution is the same as the beam width of the $2.25 \mathrm{MHz}$ ultrasound wave from the transducer; when $f_{m}=43 \mathrm{kHz}$ the resolution is very close to half of the wavelength of an $86 \mathrm{kHz}$ acoustic field, which is $1.7 \mathrm{~cm}$.

The varying resolution with modulation frequency can be explained by the different dominating sources of DF-MAET signals at different modulation frequencies. As mentioned in the Introduction section, the radiation force-generated vibration is localized around the ultrasound beam (around 1-2 $\mathrm{mm}$ at the focal zone in our case), and the DF-MAET image obtained by scanning the transducer should have a spatial resolution of the ultrasound beam width. On the other hand, the DF acoustic emission from the crystal can be best focused to half of the wavelength corresponding the difference frequency. Therefore, if the acoustic emission from the crystal is the source of the DF-MAET signal, the spatial resolution of the DF-MAET image will be much poorer than that in the case of the radiation force. As shown in Fig. 2.6 and Eq. 2.13 the radiation-force-induced vibration is inversely proportional to the DF $\delta f$, while the vibration due to acoustic emission from the crystalopt increases dramatically with the DF. Therefore, the DF-MAET resolution becomes much poorer when $\delta f$ increases.

In this experiment, the thickness of the phantom in z-direction was much smaller than the length of the focal spot, and the phantom could be treated as a 2D object. The axial resolution in DF-MAET will be addressed in Discussion section of the current Chapter. 


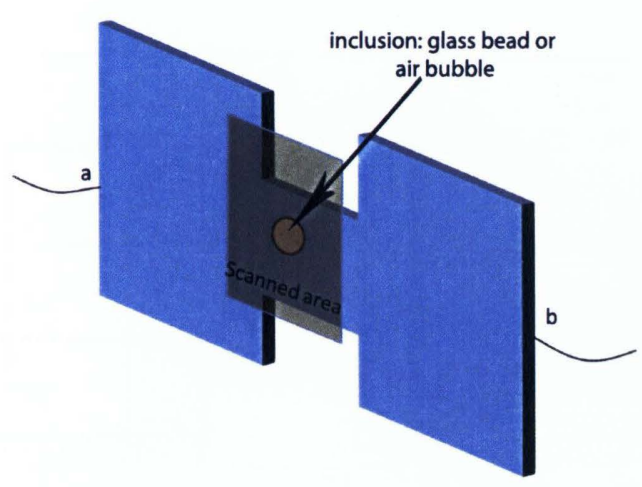

Figure 2.11: Illustration for the experiments described in section 3.3 to study the effect of the mechanical properties of the sample on the nonlinear MAET signal.

\subsubsection{Dependence of the DF-MAET signal on the acoustical prop- erties of the sample}

Interesting results were obtained for a sample including a glass bead, which had a large impedance mismatch with the surrounding gel. The acoustic impedance of glass was measured to be $z_{\text {glass }}=15.2 \mathrm{MPa} \cdot \mathrm{s} \cdot \mathrm{m}^{-1}$. The glass bead had an irregular shape and a size of around $2 \times 2 \mathrm{~mm}$. The sample was scanned two-dimensionally with a 1-mm step size across the shaded area in Fig. 2.11. The signal applied to drive the transducer was the same as that described in section 3.2. The image is shown in Fig. 2.3.3a. Fig. 2.3.3b shows the profile along the black line in Fig. 2.3.3a. It can be seen that the glass inclusion produces a larger signal $A_{\text {inc }}$ than the surrounding gelatin, $A_{\text {gel }}$; their ratio, $A_{\text {inc }} / A_{\text {gel }}$, is 1.7 .

Next, we imaged a sample including an air bubble $\left(z_{\text {air }}=412.5 \mathrm{~Pa} \cdot s \cdot \mathrm{m}^{-1}[33]\right)$. The scan has a $1-\mathrm{mm}$ step size and $f_{m}=1 \mathrm{kHz}$. The image is shown in Fig. 2.12 (a). The bubble can be clearly seen as a darker ellipsoid area. In Fig. 2.12 (b), the amplitude of the signal is also plotted across the black line in Fig. 2.12 (a) at $y=1 \mathrm{~cm}$. It can be seen that 


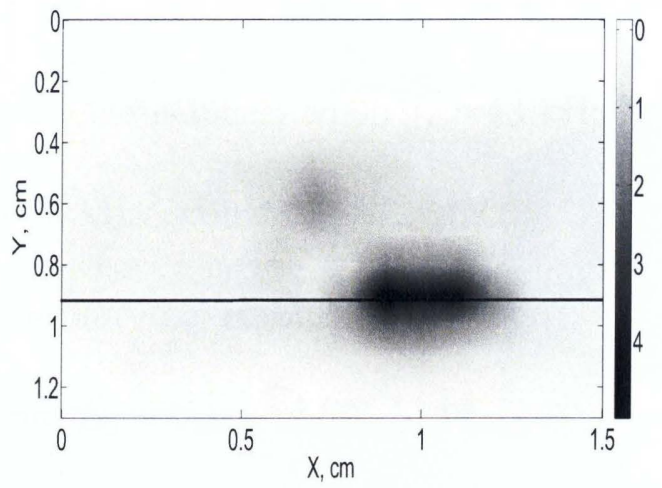

a

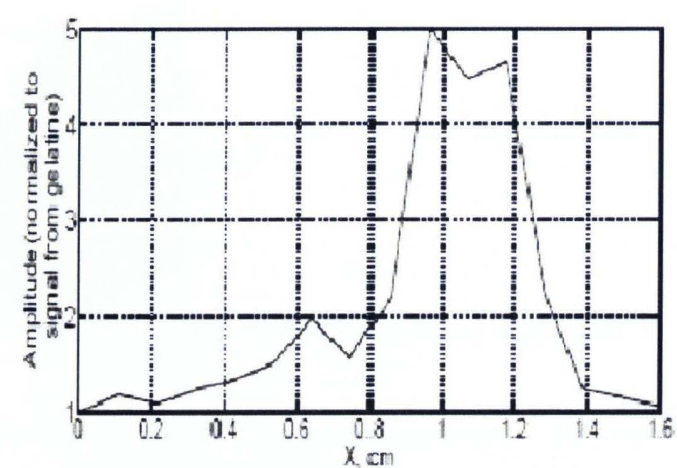

b

Figure 2.12: $1 \mathrm{~mm}$ scan of a gelatine with air bubble (a) and vertical amplitude plot along the line $\mathrm{x}=0.9 \mathrm{~cm}(\mathrm{~b})$.

the signal from the air bubble was 5 times larger than that from the surrounding gelatine. The much larger ratio $A_{\text {inc }} / A_{\text {gel }}$ for the air bubble than for the glass inclusion can be due to two factors. First, almost all of the ultrasound energy was reflected at the gelatin-air interface, generating a larger radiation force per unit area than in the case of the glass bead. Second, the part including the air bubble has a much smaller average density across the beam area than the sample with the glass inclusion and, consequently, oscillates with a larger amplitude, according to Eq. 2.12. Image of the sample with the glass inclusion (a) and the signal distribution along line $y=0.7 \mathrm{~cm}(\mathrm{~b})$.

These results confirm that the source of the observed DF-MAET signal is the radiation force when $f_{m}=1 \mathrm{kHz}$, as one can clearly see that it depends on the mechanical and acoustic properties of the inclusions in agreement with Eq. 2.12. 


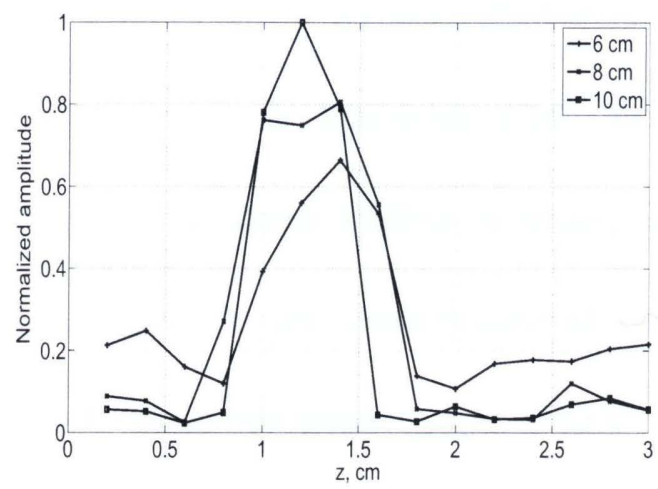

Figure 2.13: Vertical scans of the sample bridge at different distances away from transducer.

\subsubsection{Parametric effect in the medium}

In addition to the radiation force and the acoustic emission from the crystal, nonlinear acoustic effects in the medium, such as the parametric effect, can also induce vibration at the difference frequency in the sample and therefore be a potential source of a DF-MAET signal. For a focused transducer, vibrations due to the parametric effect are much stronger around the focal zone than the pre-focal area. To examine this phenomenon, we performed a set of vertical scans of the 7 -mm wide bridge in a sample similar to that depicted in Fig. 2.8 at different distances from the transducer with a scanning step of $2 \mathrm{~mm}$. The results of the scans are shown in Fig. 2.13 for distances of 6, 8 and $10 \mathrm{~cm}$ from the transducer. Again, the focal length of the transducer was $10.2 \mathrm{~cm}$. We observed that even at locations before the focal zone, such as 6 and $8 \mathrm{~cm}$ away from the transducer, we still obtained sharp images, which can be deduced from the FWHMs of the profiles.

This part of the experimental results proves that the nonlinearity in the medium does not contribute much to the DF-MAET signal and may be neglected. If the DF-MAET signal is to a large extent due to the parametric effect in the oil, we should have observed 
a peak of much larger full-width-half-maximum at 6 and $8 \mathrm{~cm}$. That is because a nonlinear DF pressure field arising in the focal zone will diverge and have a much smaller amplitude. Also, its corresponding beam diameter will be much larger than that in the focal zone at the locations of 6 and $8 \mathrm{~cm}$ from the transducer. Yet, the experiment shows that the maximum of the amplitude of the DF-MAET signal and FWHM of the image at $10 \mathrm{~cm}$ is comparable with those at 6 and $8 \mathrm{~cm}$. Therefore, we can conclude that the DF-MAET signal is not caused by the parametric effect.

\subsection{Discussion}

The study presented in this Chapter demonstrates the possibility of using the radiation force in DF-MAET. However, several potential improvements may be made to the present experimental setup.

First, in our preliminary DF-MAET experiments, the lateral spatial resolution is defined by the beam width (about $2 \mathrm{~mm}$ ) at the focus, but the axial resolution is much poorer due to the millisecond duration of the burst. If a transducer produces a burst with a duration of $\tau_{p}$, then the axial resolution is given by $l_{p}=v_{s} \tau_{p}$, where $v_{s}=1.5 \mathrm{~mm} / \mu \mathrm{s}$ is the speed of acoustic waves in water or soft tissues. One potential method to improve the axial resolution is to use the static radiation force generated by a short microsecond ultrasound pulse rather than by a millisecond ultrasound burst with two frequencies, because the short pulse can provide a good axial resolution apparently equivalent to the spatial extent of the pulse. We used the dynamic radiation force configuration in our initial DF-MAET experiments because it gave better SNR. Yet, because of the low frequency of the differential MAET signal, we had to 
use long bursts of several ms, which cost us axial resolution. Another potential method to improve the axial resolution is to create a localized DF vibration by applying two transducers that are separated angularly. Their acoustic fields would overlap only at the intersection of the two beams. The frequencies $\omega_{a}$ and $\omega_{b}$ fed to each transducer should be slightly different, and the DF-MAET signal generated at the intersection would have a frequency of $\left|\omega_{a}-\omega_{b}\right|$.

Second, as we fed the driving signal with two different frequencies to a single element transducer, the nonlinear effects in the piezo crystal lead to the emission of DF acoustic waves by the transducer, as shown in Fig. 2.6 and Fig. 2.7. We also showed that the emission of DF acoustic waves gradually becomes the dominating source of the DF-MAET signals and produces DF-MAET images with poor spatial resolution with the increase of the difference frequency. Therefore, it is desirable to reduce the emission of DF acoustic waves as much as possible. Again, the configuration of two angularly separated transducers should be able to solve this problem. However, it would be technically challenging to focus two transducers to one point. An alternative to the configuration of the two angularly separated transducers would be a two-element transducer, as used in VAG by Fatemi and Greanleaf [35]. Such a transducer consists of two concentric elements operating at two slightly different frequencies. The total acoustic field in this case exerts a dynamic radiation force at the difference frequency. The DF acoustic emission would be minimized as a single frequency is applied to each element. Such a dual-element transducer would also solve the problem of the low axial resolution as the beams from concentric elements would overlap only around the focal zone of the transducer. This would improve the axial resolution to the length of the focal zone.

Lastly, we will discuss safety considerations due to the use of ultrasound. With the en- 
ergy conversion efficiency of $40 \%$ for the transducer used in our experiments, $20 \mathrm{~W}$ of $50 \mathrm{~W}$ of applied power were actually transferred to acoustic waves. For a $2.25 \mathrm{MHz}$ transducer, the focused beam area is estimated to be $4 \mathrm{~mm}^{2}$. Thus, the intensity at the focal zone was estimated to be $370 \mathrm{~W} / \mathrm{cm}^{2}$, which is far above the safety limit. The DF-MAET signals, however, could still be detected at an effective power as low as $2 \mathrm{~W}$, corresponding to an intensity of $37 \mathrm{~W} / \mathrm{cm}^{2}$ at the focal zone. However, weaker MAET signal would certainly reduce the SNR and, consequently, the image quality. The potential methods to use ultrasound with the intensity below the safety limit to generate DF-MAET with sufficient SNR include using a stronger magnetic field, such as the $1.5 \mathrm{~T}$ magnetic field in clinical MRI and reducing the noise level in our detection system.

It should be pointed out that the DF-MAET image provides both the information on the mechanical and electrical contrast of the sample. As shown in Eq. 2.1, the DF-MAET signal depends on both $\mathbf{v}$ and lead field current density $\mathbf{J}_{\mathbf{a b}}$. While DF vibration velocity $\mathbf{v}$ depends on the mechanical properties of the sample, $\mathbf{J}_{\mathbf{a b}}$ depends on the electrical properties of the sample. To reconstruct the electrical conductivity of the sample, we need to extract $\mathbf{J}_{\mathbf{a b}}$ from the DF-MAET signal. One possible solution is to combine VAG with DF-MAET. The DF-MAET signal can be normalized to the VAG signal to eliminate the effect of the heterogeneity of the mechanical properties of the sample.

It was mentioned above that, like any imaging modality making use of the focused ultrasound, DF-MAET has a lateral resolution limited by the beam diameter ( $2 \mathrm{~mm}$ ). The axial resolution is limited by the length of the focal zone. At the same time, the applications of such modalities as MRI and Computed Tomography for microscopy applications and precise 3D reconstruction reached sub-micron resolution. 


\subsection{Conclusion}

We were able to apply the radiation force to MAET. High spatial resolution images were formed by scanning the focal point across gelatine samples with $f_{m}=1 k H z$. We have shown that, at low difference frequencies, the DF-MAET signal is mainly due to the radiation force. When $f_{m}$ increases, the acoustic emission from the transducer increases drastically and ultimately dominates the DF-MAET signal, resulting in low-spatial-resolution images. We also investigated how the DF-MAET signal is dependent on the mechanical properties of the sample, and the results agree with the prediction from the simplified model of the dynamics of the sample. 


\section{Chapter 3}

\section{The possibility of imaging}

\section{permittivity of biological tissues with}

\section{ultrasound}

3.1 Magnetic moment induced by applying ultrasound to a polarized tissue

\subsubsection{Principle}

The dielectric constants of biological tissues are significantly large. For example, muscle tissue has the dielectric constant of $1.8 \times 10^{7}$ (at $10 \mathrm{~Hz}$ ), while whole blood has one of $5.3 \times 10^{3}($ at $1 \mathrm{kHz})$, which is much larger than, for example, water $\left(\epsilon_{r}=80\right)[3]$. The difference in $\epsilon_{r}$ between biological tissues and blood suggests that highly vascularized areas, 


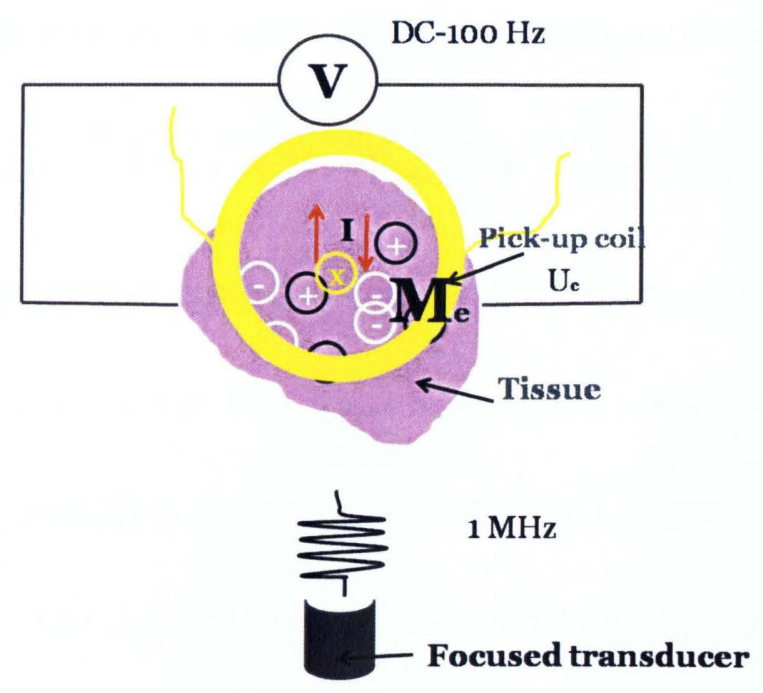

Figure 3.1: Schematic of the setup to detect the signal from a biological tissue through polarization.

say, malignant tumors, will have $\epsilon_{r}$ much lower than that of normal tissues. We will first show that a magnetic moment can be induced by an ultrasound propagating through a polarized dielectric. Then, we will derive the formula for the signal amplitude. Lastly, we will demonstrate the experimental results for detection of the magnetic moment with a pick-up coil and probe electrodes [42].

Consider Fig. 3.1. A low-frequency (DC-100 Hz) AC voltage source is applied to a biological sample through a pair of electrodes. A polarization will occur in the tissue; that is, charges will accumulate either in the vicinity of the cell membrane and create cell-size dipoles. These separated charges are schematically shown in Fig. 3.1. Next, an acoustic pulse of much higher frequency as compared with the voltage source (say, $1 \mathrm{MHz}$ ) is applied to the sample. From the 'viewpoint' of the ultrasonic pulse, the charge separation looks quasistatic. The acoustic vibration will cause the oscillation of the medium and of the charges. Two opposite local currents will associate with this movement. Lastly, these currents will form a magnetic 
moment oscillating at the ultrasound frequency. The time-varying magnetic moments can be picked up by either a coil or probe electrodes $a$ and $b$ shown in Fig. 3.1.

\section{Theory behind magnetic moments induced by an ultrasound passing through a polarized dielectric sample}

It has been derived in [1], that for a sample with a distribution of electric field $\left(\mathbf{E}_{\mathbf{1}}\right)$ and the lead field for the given probe electrode configuration $\mathbf{J}_{\mathbf{a b}}$ the voltage detected by probe electrodes can be found as:

$$
V_{m}=\int_{\Omega} \mathbf{J}_{\mathrm{ab}} \cdot \mathbf{E}_{1} \mathrm{dr} .
$$

Now, we can consider a dielectric where the charge separation occurs in an electric field (see Fig. 3.6). With the charge movement induced by the ultrasound, we can asssociate the current density and the electric field for both positive and negative charges:

$$
E_{+}=\frac{n e \mathbf{v}}{\sigma}, E_{-}=\frac{n e \mathbf{v}}{\sigma} .
$$

Substituting these quantities in Eq. 3.14 yields:

$$
\begin{aligned}
V_{m} & =\int_{\Omega}\left(\frac{n e \mathbf{v}}{\sigma_{+}} \cdot \mathbf{J}_{\mathbf{a b}+}-\frac{\mathbf{n e v}}{\sigma_{-}} \cdot \mathbf{J}_{\mathbf{a b}_{-}}\right) \mathbf{d} \mathbf{r} \\
& \left.=\int_{\Omega}\left(\frac{n e}{\sigma} \mathbf{v}\right)\left(\mathbf{J}_{\mathbf{a b}+}-\mathbf{J}_{\mathbf{a b}_{-}}\right)\right) \mathbf{d r} \\
& =\int_{\Omega} \frac{n e}{\sigma} \mathbf{l}_{\mathbf{p}} \cdot \nabla_{\mathbf{J}}\left(\mathbf{E}_{\mathbf{a b}} \cdot \mathbf{v}\right) \mathbf{d} \mathbf{r} \\
& =\int_{\Omega}\left(\mathbf{P} \cdot \nabla_{\mathbf{J}}\left(\mathbf{E}_{\mathbf{a b}} \cdot \mathbf{v}\right)\right) \mathbf{d r},
\end{aligned}
$$

where $\nabla_{J}$ means the spatial differential is carried on $\mathbf{J}_{\mathbf{a b}}$ or $\mathbf{E}_{\mathbf{a b}}$. Further, we will use the following identity to expand the function under integral:

$$
\nabla(\mathbf{A} \cdot \mathbf{B})=\mathbf{A} \times(\nabla \times \mathbf{B})+\mathbf{B} \times(\nabla \times \mathbf{A})+(\mathbf{A} \nabla) \mathbf{B}+\mathbf{B}(\nabla \mathbf{A})) .
$$


If we assume the velocity $v$ to be constant, only two terms in the integral will be left:

$$
V_{m}=\int_{\Omega}\left(\mathbf{P} \cdot\left(\mathbf{v} \times \nabla \times \mathbf{E}_{\mathbf{a b}}\right)+\mathbf{P} \cdot\left(\mathbf{v} \cdot \nabla \mathbf{E}_{\mathbf{a b}}\right)\right) \mathbf{d r}
$$

In case of the detection with electrodes, only the second term will be non-zero, while in case of the detection with a coil the first term will dominate.

The definition of the magnetic moment of an arbitrary current density is:

$$
\mathbf{M}=\int_{\Omega}(\mathbf{r} \times \mathbf{J}) \mathbf{d} \mathbf{r}
$$

In case of 2 current distributions associated with the displacement of charges in ultrasound field, $\mathbf{J}_{\mathrm{ab}+}$ and $\mathbf{J}_{\mathrm{ab}_{-}}$, we have

$$
\begin{aligned}
\mathbf{M} & =\int_{\Omega}\left(\mathbf{r}_{+} \times \mathbf{J}_{+}+\mathbf{r}_{-} \times \mathbf{J}_{-}\right) \mathbf{d} \mathbf{r} \\
& =\int_{\Omega}\left(\mathbf{r}_{+} \times \mathbf{J}-\mathbf{r}_{-} \times \mathbf{J} \mathbf{d} \mathbf{r}\right) \\
& =\int_{\Omega} \mathbf{J} \times \mathbf{l}_{\mathbf{p}} \mathbf{d r} \\
& =\int_{\Omega} n q \mathbf{v} \times \mathbf{l}_{\mathbf{p}} \mathbf{d r} \\
& =\int_{\Omega} n q \mathbf{v} \times \mathbf{l}_{\mathbf{p}} \mathbf{d r} \\
& =\int_{\Omega} \mathbf{v} \times \mathbf{P} \mathbf{d} \mathbf{V}
\end{aligned}
$$

Thus, the final formula for the voltage in the coil will be:

$$
U_{m}=\omega \int_{\Omega} \mathbf{B}_{\mathbf{r}} \cdot \mathbf{M}(\mathbf{r}, \mathbf{t}) \mathbf{d r},
$$

where $\mathbf{B}_{\mathbf{r}}$ is the magnetic field generated by the pick-up coil when a unit current goes through it. 


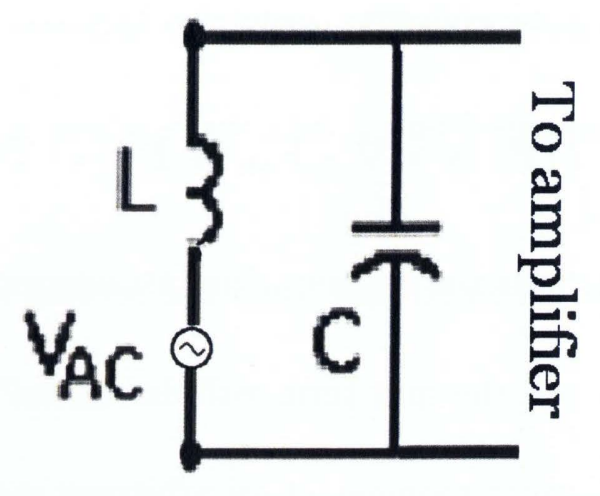

Figure 3.2: Schematic representation of a pick-up coil connected to amplifier.

\subsubsection{Experimental setup and results: detection with coil}

\section{Design of the detection coil}

From the Eq. 3.7, one can see that $\mathbf{B}_{\mathbf{r}}$, a magnetic field created by the coil when $1 \mathrm{~A}$ of current is passed through it, needs to be as large as possible. A coil can be electrically presented as an inductor and a capacitor connected in parallel (Fig. 3.2). The capacitor represents the coil's own stray capacitance and the input capacitance of the following amplifier.

The detection of the magnetic moment described here has some similarity to the signal described detection in MRI. In both cases, RF coils are used to detect induced magnetic moments However, the signal detection in MRI has an important difference due to the difference in bandwidth. In MRI, the signals have a very narrow bandwidth. Therefore, the coils are working at resonance frequency to increase the sensitivity. In our experiments, to obtain a good spatial resolution, ultrasound signals have broad bandwidth. Therefore, we also need the resonance frequency of the coil to be above the signal spectrum to detect the broad band signal. If the coil's resonance frequency is below the band of the signal, the coil behaves like a capacitor shunting the voltage dropping on the inductance. 


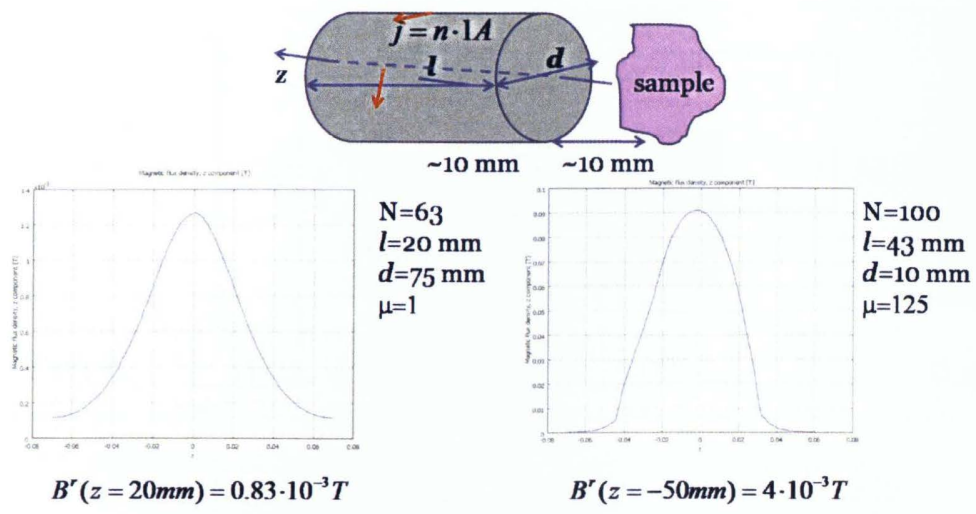

Figure 3.3: Model used in simulations and $B_{r}$ distributions for two coils.

The value for $\mathbf{B}_{\mathbf{r}}$ for a particular coil was numerically calculated in a Comsol model provided by A. Hrbek. The idea along with the simulated graphs for the $\mathbf{B}_{\mathbf{r}}$ is as following.

The input parameters were the length, diameter and density of turns of the coil. It was assumed that the coil's core was air $(\mu=1)$ or ferromagnetic $(\mu=125)$ to enhance the magnetic flux density. The result is the distribution of $\mathbf{B}_{\mathbf{r}}$ in space and in particular along the coil's axis. We were intereseted in values of $\mathbf{B}_{\mathbf{r}}$ one $\mathrm{cm}$ away from the center of the coil, as the coil was supposed to be places on top of the sample. We used our results to estimate the voltage signal we could expect.

\subsection{Conductivity and relative permittivity of a muscle}

\section{tissue}

First, we studied the electrical properties of the pork muscle tissue used in our experiments in a sub-kHz range of frequencies. Both specific conductivity and relative permittivity of a muscle tissue were measured in the experiments. 

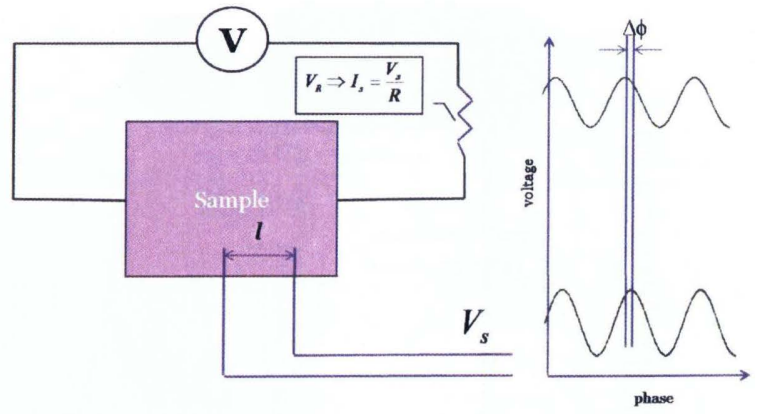

Figure 3.4: Illustration of the experiments to measure specific conductivity and relative permittivity of a muscle tissue.

We used 4-electrode method to measure the electrical properties (Fig. 3.4). AC voltage $\mathrm{V}$ was applied to the sample in series with a $1 k \Omega$ resistor through a pair of electrodes. The current in a system was detected through voltage across the resistor (sine wave on top of the graph). The voltage across the sample was measured through another pair of electrodes and detected simultaneously. Due to capacitive properties of the muscle tissue, there was a phase difference between the current and the voltage across the sample. This phase difference, $\Delta \phi$, was found with in $M A T L A B^{T M}$ from the cross correlation between the voltage and the current in time domain.

Next, we considered the two parts of the complex admittivity

$$
\gamma=\sigma+i \epsilon_{0} \epsilon_{r} \omega, \sigma=\gamma \cos \Delta \phi, \epsilon_{r}=\frac{|\gamma| \sin \Delta \phi}{\epsilon_{0} \omega}
$$

$|\gamma|$, on the other hand is $\frac{1}{z_{s}}$, where $z_{s}=Z_{s} s / l$ is the impedivity of the sample, and $Z_{s}=\frac{V_{s}}{I_{s}}$ is the absolute value of the impedance of the sample; $V_{s}$ is the voltage between the probing electrodes and $I_{s}$ is the current in the circuit. The results of these measurements are presented in Table 3.1.

We observed that the conductivity of the sample remained constant, $0.08 \mathrm{Sm} \cdot \mathrm{m}^{-1}$ within 
Frequency, $\mathrm{Hz} \quad 10 \quad 50 \quad 100 \quad 500 \quad 10$ (Miklavcic et al, 2006)
$\sigma, S m \cdot m^{-1}$
0.08
0.08
0.08
0.08
$0.04-0.14$

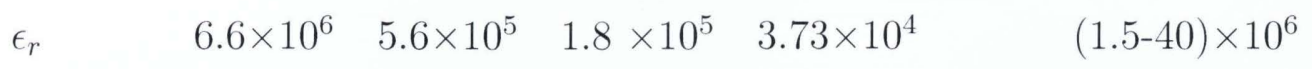

Table 3.1: Measured values of $\epsilon_{r}$ and $\sigma$ for a slub of pork muscle tissue at several frequencies. the frequency range of $10-500 \mathrm{~Hz}$, while the relative permittivity decreased by two orders, which agrees with the known data on the dispersion of relative permittivity of bilological tissues. Both $\epsilon_{r}$ and $\sigma_{r}$ for muscle tissue are in good agreement with data from [?]. These results will be used in experiments and estimations of the magnitude of signals described below.

\section{Signal estimation}

The magnitude of polarization $\mathbf{P}$ can be found as $\epsilon_{r} \epsilon_{0} U_{0} / d$, where $U_{0}$ is the applied voltage, and $d$ is the distance between the source eletrodes. If $A$ is a gain of the detection system, we have

$$
U_{m} \approx \omega B_{r} v \epsilon_{r} \cdot \epsilon_{0} \cdot U_{0} \cdot V \cdot A / d
$$

Here, $V$ is the volume where the magnetic moment is generated. Assuming: $\omega=6.28 \times$ $10^{6} \mathrm{~s}^{-1}, B_{r}=10^{3} \mathrm{~T}, v=1 \mathrm{~m} / \mathrm{s}, \epsilon_{r}=10^{7}, E=30 \mathrm{~V} / 2 \mathrm{~cm}=1500 \mathrm{~V} / \mathrm{m}$ (far above the safety limit), $V=10 \mathrm{~mm}^{3}$ and amplification of 300 times, we get $U_{m}=0.05 \mathrm{mV}$. 


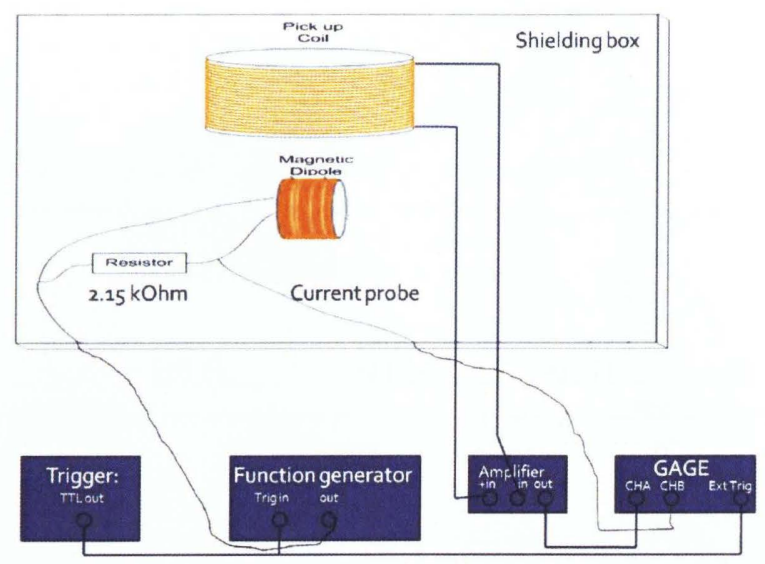

Figure 3.5: Illustration of the experiments to measure coil's sensitivity.

\section{Coil's sensitivity}

We verified the coil's sensitivity using a magnetic moment of a known value, which was another small coil with a known current passing through it. The scheme for these experiments is shown on Fig. 3.5.

In this experiment, the signal generated in the coil was measured twice, when the magnetic moment was oriented parallel and perpendicular to the coil. $1 \mathrm{MHz}$ voltage signal was applied to the small coil in series with a resistor. The current probe read the voltage off the resistor. The signal form the coil was amplified 300 times. When the magnetic moment was perpendicular to the coil, we could only observe the noise and disturbance from the source function generator. The estimation was done as following. Form Eq. 3.7, we have

$$
V_{0}=\omega_{r} m_{0} \cdot \text { gain }
$$

The magnetic moment is simply

$$
m_{0}=I N \pi D^{2} / 4
$$

A pick-up coil with the following parameters was used: 
Length $=1 \mathrm{~cm}$

Diameter $=7 \mathrm{~cm}$

Number of turns $=25$

$\mathrm{Br}=0.23 \mathrm{mT}$.

Magnetic moment was found substituting following parameters:

$\mathrm{N}=5$

Diameter $=1.3 \mathrm{~cm}$

$\mathrm{I}=70 \mu \mathrm{A}$

And, for the sensitivity, we used $V_{1}$, voltage generated in the coil when the magnetic moment was parallel to the coil, $V_{2}$ is that when the magnetic moment was perpendicular to the coil:

$$
\eta=\frac{V_{1}-V_{2}}{V_{0}}=1.12 .
$$

The fact that the sensitivity $\eta$ was larger than 1 , can be due to inaccurate gain of the amplifier used. The result suggested that the sensitivity of the coils could be taken for 1 .

\section{Experimental setup and results}

The experimental setup for the detection of the signal with coil is shown on Fig. 3.6.

We designed following signal to trigger the data acquisition card (DAQ). We want to trigger the transduser at the maximum of the source voltage but we need high repetition rate as well. The function generator 1 acts as a source which applies $\mathrm{AC}$ voltage to the sample. Its synchronization output produces square pulses at the same frequency which drive function generator 2 . The function generator 2 produces short pulses of 10 ms duration and repetition rate of $10 \mathrm{~Hz}$. It triggers pulse generator 1 which in turn produces very short 


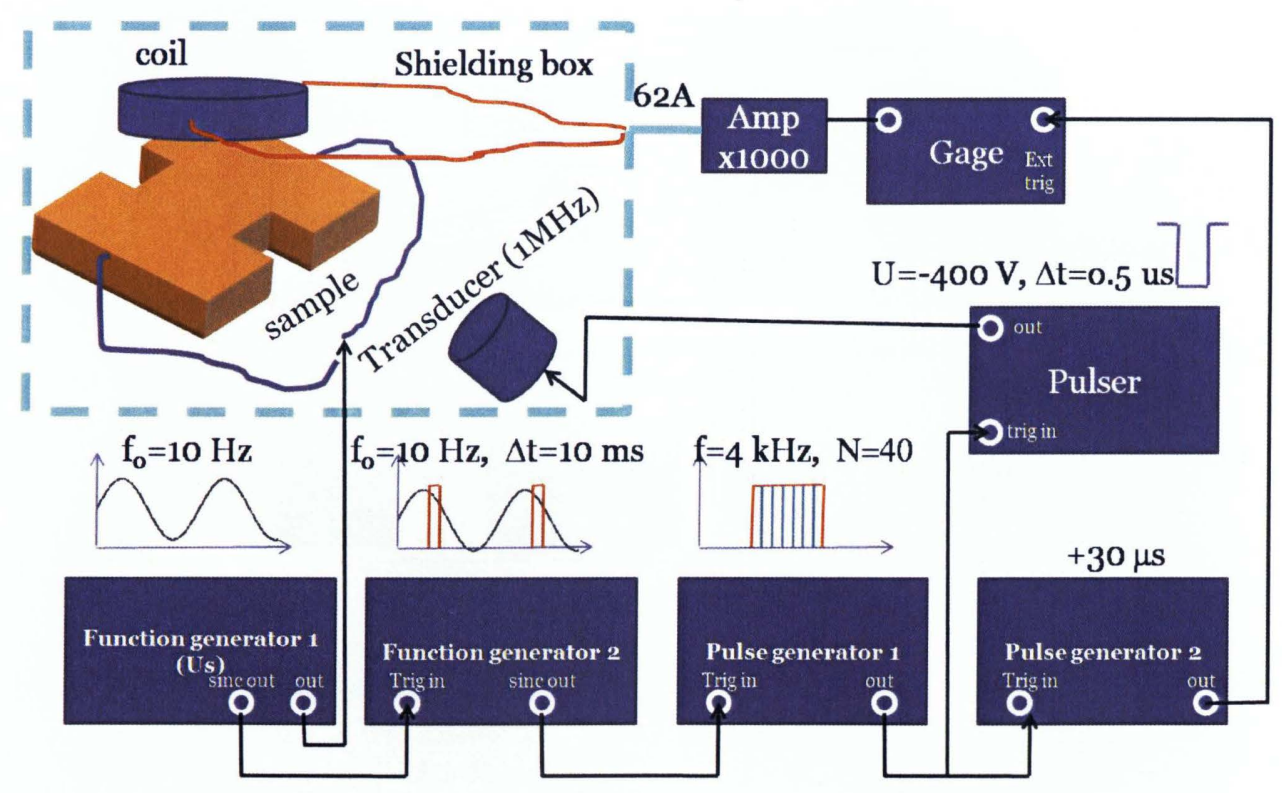

Figure 3.6: Experimental setup to detect the signal with coil.

pulses separated by 250 us. This is the signal driving the pulser. Pulser produces square voltage signal at $-400 \mathrm{~V}$, of $25 \mathrm{~ns}$ duration, at central frequency of $1 \mathrm{MHz}$. The transducer emits a pulse, which is focused at the sample generating magnetic moment in the focal zone. Signal is then detected with a coil, amplified and digitized in the Gage card. To avoid capture of the initial disturbance, the Gage card is triggered 20 us later after pulser. The delay is created in pulse generator 2. The result is shown on Fig. 3.7, with the lowest noise level $(0.01 \mathrm{mV})$ reached for reasonable averaging.

Recalling the estimated value for the signal of $0.05 \mathrm{mV}$, one can expect SNR of 5 for the noise level reached. Yet, there was evidence of the signal present. Then, we switch to the detection of the signal with electrodes. 


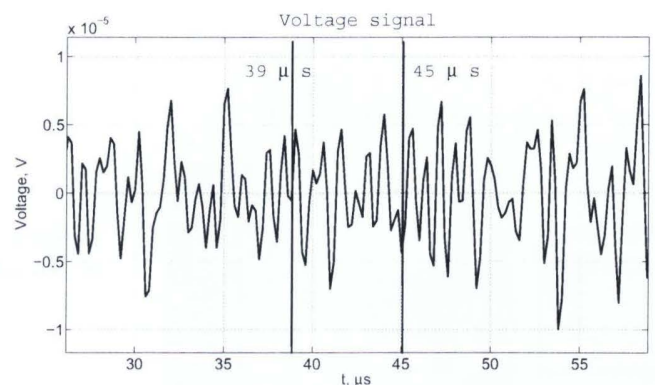

a

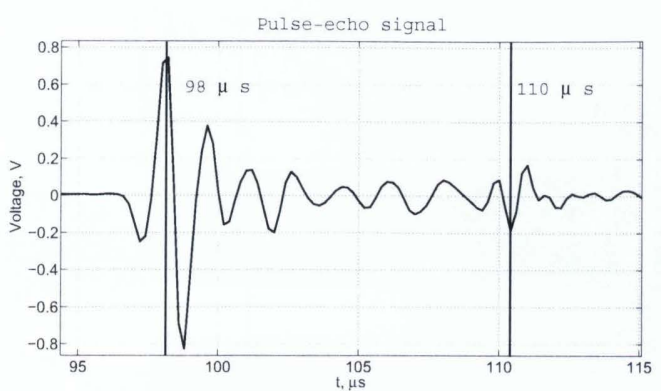

$\mathrm{b}$

Figure 3.7: The signal from the pickup coil in time domain. The signal was expected at 35 and $41 \mu s$ (a), corresponding to the interfaces indicated by the bursts in pulse-echo signal (b).

\subsection{Detection of the magnetic moment with electrodes}

\subsubsection{Sample and experimental setup}

The measurements were done on a sample of pork shown in 3D and front views on Fig. 3.8. The top plane of the sample was normal to the direction of the muscle fibers. The sample was cut in a П-shape in order to bring electrodes as far back as possible from the transducer. This was done in order to eliminate the disturbance when the ultrasound hits the electrodes, or, in other words, to delay the disturbance in time and make the acousto-electric signal clearly distinguishable.

The realization of the experiment is shown on Fig. 3.3.1. The sample is immersed in the coupling oil. The oil was preferred to water due to its minimal conductivity, because the external current would cause the disturbances all throughout the captured time domain. The transducer was focused at the narrow bridge, where the current density is higher. This would allow obtaining a larger voltage signal, as it is proportional to the lead field current 


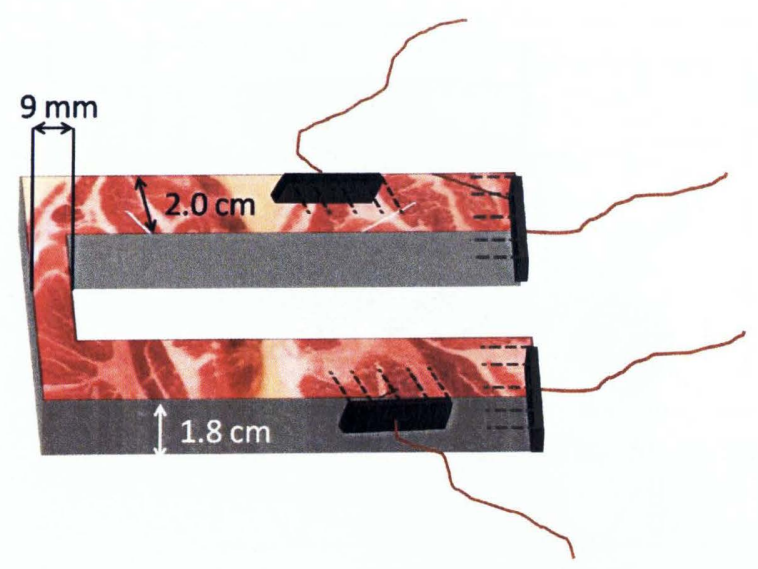

a

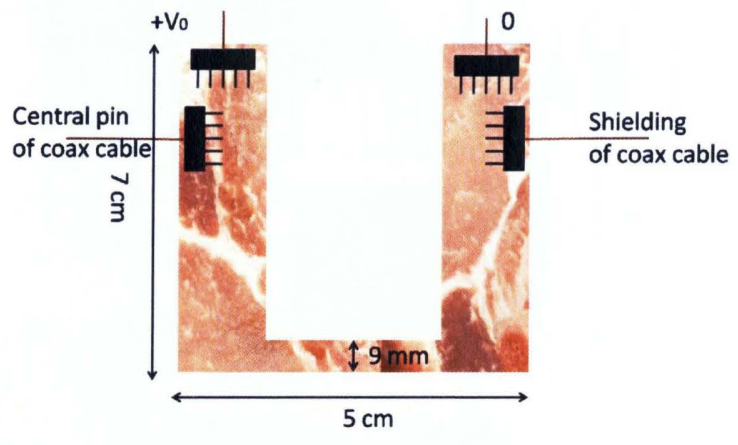

b

Figure 3.8: Sample used for studying the voltage signal

density. The applied DC voltage was generated by the series of 4 batteries (Energizer Canada Inc.). The pulser was used to feed the transducer with an ultrashort pulse with the temporal duration of half of the period corresponding to the central frequency of the transducer (1 $\mathrm{MHz}$. The transducer was wrapped in a piece of aluminum foil connected to the shielding box to further reduce the disturbance caused by the electromagnetic emission from the transducer. The signal was collected with the probe electrodes both seen on Fig. 3.8 and 3.3.1, passed through $1 \mathrm{MHz}$ low-frequency filter, an RC circuit specifically designed for the experiments. For signal amplification, we used a 300-times Panametrics NF SA-420F5 amplifier for the first stage, as well as SR 560 preamplifier giving a second stage amplification of 20 times and also providing $10 \mathrm{kHz}$ high-pass filtering. The amplified and filtered voltage signal from the pork sample was digitized by the Gage card. The Gage card was triggered with a time delay of $\tau=20 \mu s$, to prevent the overload of the Gage card. Pulse-echo signal was recorded simultaneously in the second channel of the data acquisition card. Tools and functions of $M A T L A B^{T M}$ (Mathworks Inc.) were used to manipulate and filter the digitized 


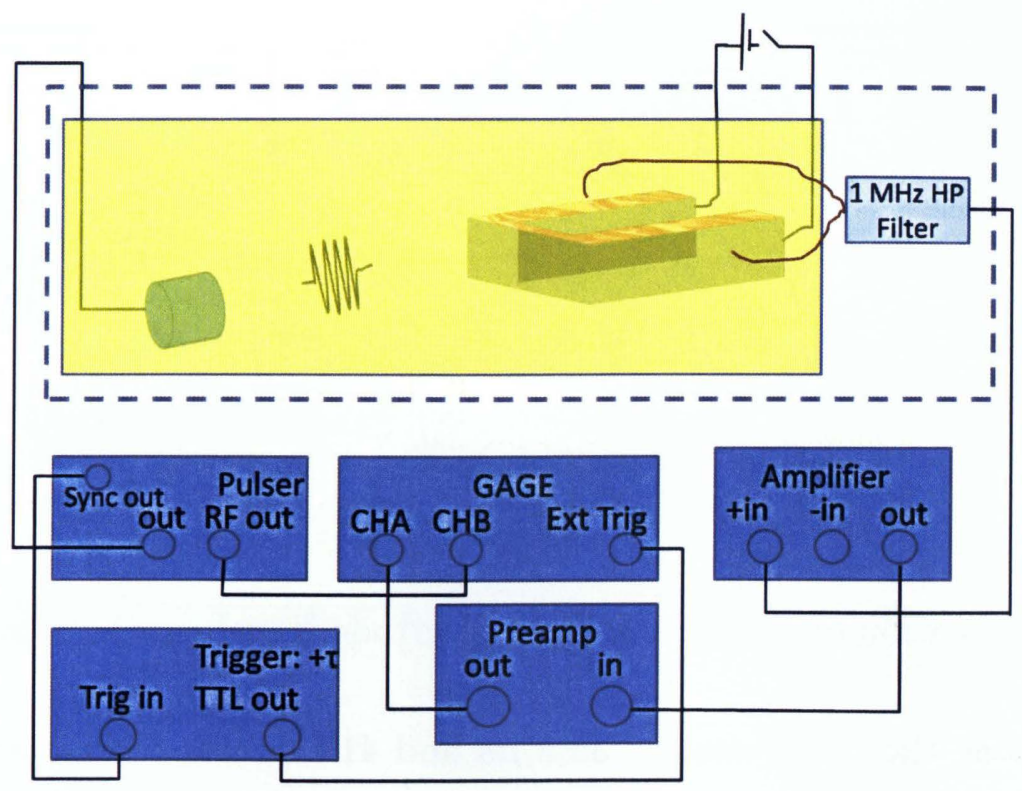

Figure 3.9: Illustration of the signal detection with electrodes.

signals.

\subsubsection{Results}

First, we verified that the voltage signal we observe indeed originates in the pork sample.

We acquired both pulse-echo and voltage signals when the transducer was located at two different distances away from the sample: $8.1 \mathrm{~cm}$ and $9.5 \mathrm{~cm}$. The pulse-echo and electrode signals are shown on Fig. 3.10a and 3.10b, respectively. The time delay $\tau$ was $20 \mu s$. The voltage data were acquired with the amplification of 3000 times, sampling rate of $5 \mathrm{MHz}$, averaging of $5 \times 10^{4}$ times, DC voltage of $32 \mathrm{~V}$.

Calculated from the pulse-echo data on Fig. 3.10, the signal from electrodes was expected in between 39 and $44.9 \mu \mathrm{s}$ for $d_{1}=8.1 \mathrm{~cm}$ and in between 43.5 and $49.15 \mu \mathrm{s}$ for $d_{2}=9.5 \mathrm{~cm}$. The experimental electrode signal on Fig. 3.10b is in good agreement with those estimations. 


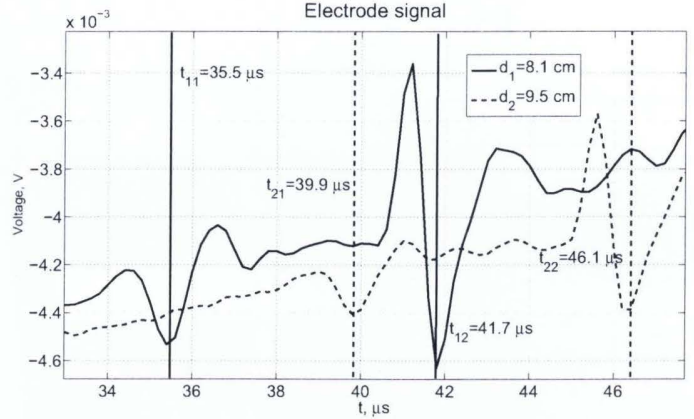

a

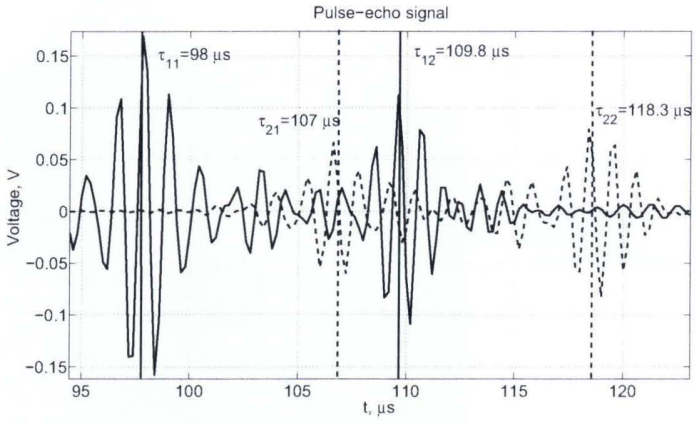

b

Figure 3.10: Verification of the fact that the electrode signal comes from the pork muscle

Indeed, one can see the two pulses at $35.5 \mu \mathrm{s}$ and $41.7 \mu \mathrm{s}$ for the distance $d_{1}$ (solid line) and at $39.9 \mu \mathrm{s}$ and at $46.1 \mu \mathrm{s}$ for the distance $d_{2}$ (dashed line). It is clear that only the two bursts are observable at the interfaces between the muscle and the oil. In both cases, the bursts are separated by $\Delta t=6.2 \mu \mathrm{s}$, which is exactly what it takes for an ultrasonic pulse to travel $0.9 \mathrm{~cm}$ in tissue (assuming the speed of sound in muscle $1500 \mathrm{~m} / \mathrm{s}$ ).

The electrode signals on Fig. 3.11c and 3.11e refer to the different polarities of the applied voltage. It is obvious that the detected voltage signal also changes the polarity.

\section{Electrode signal in absence of applied voltage}

We also were able to detect the signal from electrodes without the applied DC voltage. For this experiment, we kept the parameters same as before except for the DC voltage $V_{0}=0 \mathrm{~V}$ and averaging of $5 \times 10^{5}$ times. Again, the pulse-echo and the voltage signal were detected simultaneously. The results are shown on Fig. 3.11.

Fig. $3.11 \mathrm{a}$ and $\mathrm{b}$ refer to the absence of the applied voltage. The pulse-echo signal indicated that the ultrasonic pulse faces the 1st interface at $118 \mu \mathrm{s}$, which would correspond to $39 \mu s$ for the electrode signal. Indeed, we were able to detect a pulse starting at $40 \mu s$. The 


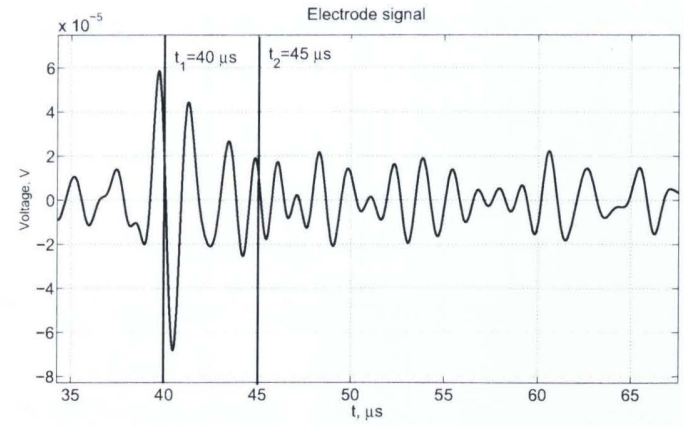

a

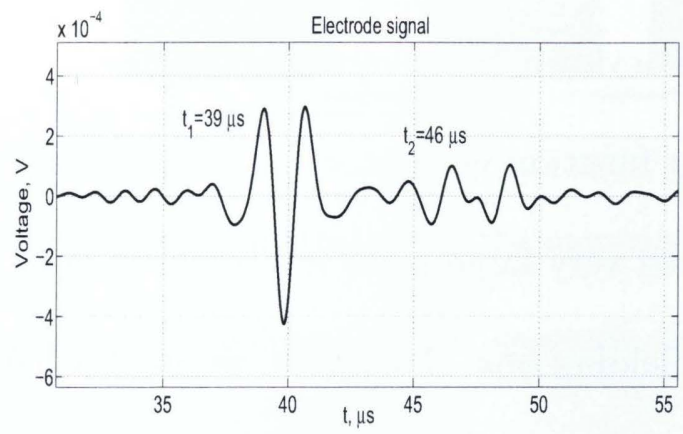

C

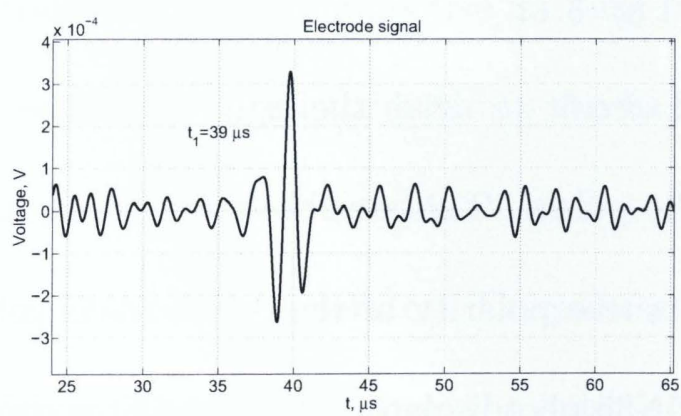

$\mathrm{e}$

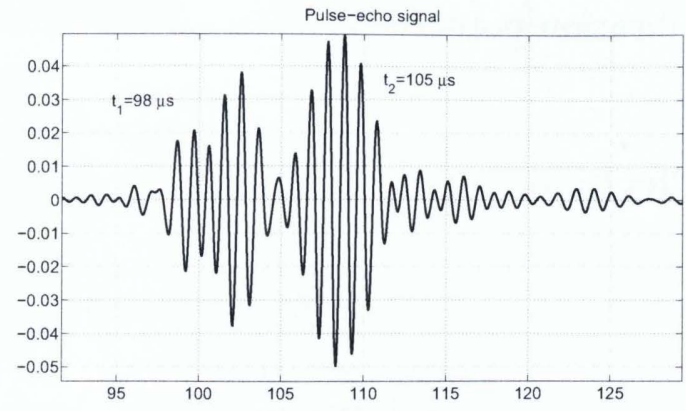

$\mathrm{b}$

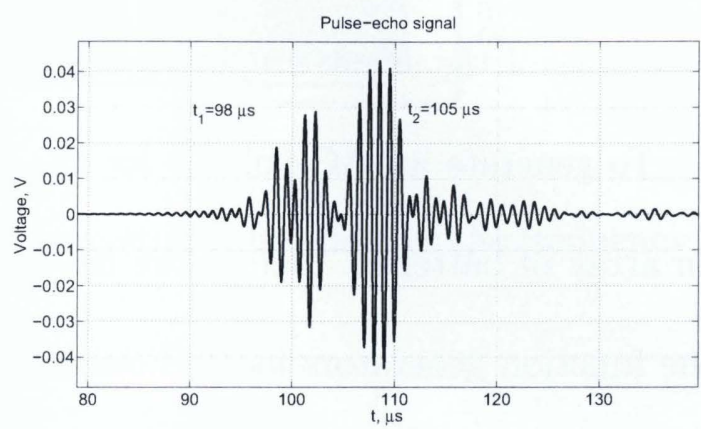

d

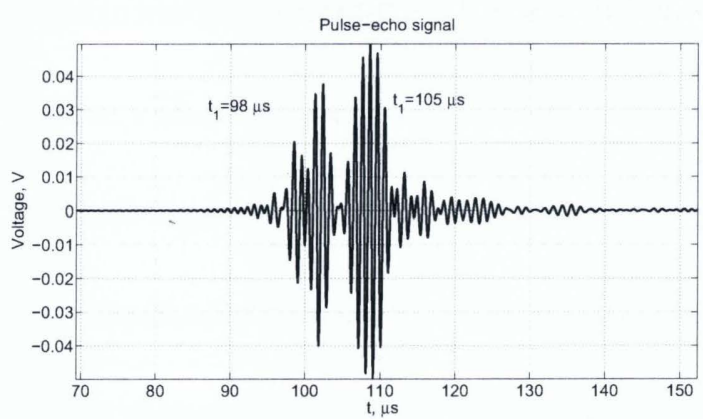

f

Figure 3.11: Experiments on the pork sample to verify the effect of the polarity and the electrode signal in absence of the external voltage. 
pulse corresponding to the rear boundary could not be distinguished.

The electrode signal generated by the ultrasound in our sample was due to ultrasonic vibration potentials. More results on UVP will be presented in the following chapter.

\section{Electrode signal for different frequencies of the applied voltage}

Next, we checked if the electrode signal is related with the polarization inside the tissue. As the tissue's dispersive nature leads to the dispersion of $\epsilon_{r}$ with high increments, the connection between the electrode signal and the polarization should be noticed as certain dynamics of the signal amplitude with the frequency of applied voltage.

To generate an $\mathrm{AC}$ voltage for the sample, a function generator was needed instead of an array of batteries. Before, we have experienced very large noise levels which came from the function generators located outside of the shielding box. Therefore, we used a batteryoperated function generator (Medi Cal, Model 220) which was placed inside the shielding box. The whole experimental setup is shown on Fig. 3.12.

In this case, we designed a special electrical circuit to drive the signal from the Medi Cal function generator through the system to the pulser. This was done to make sure the transducer only fires while half-period of the positive polarity of the applied AC voltage. This feature was realized through the use of the 4 N35 photoisolator. A optoisolator contains a light emitting diode (LED) and a transistor with no direct electrical contact between them. When a current is applied to the LED, its light makes the base of the transistor conductive. By applying the voltage to the base, we can modulate the current in the transistor, and pick up a voltage of a desired amplitude between the emitter and the collector.

We applied the voltage from the standalone function generator to the LED of the 4 N35 


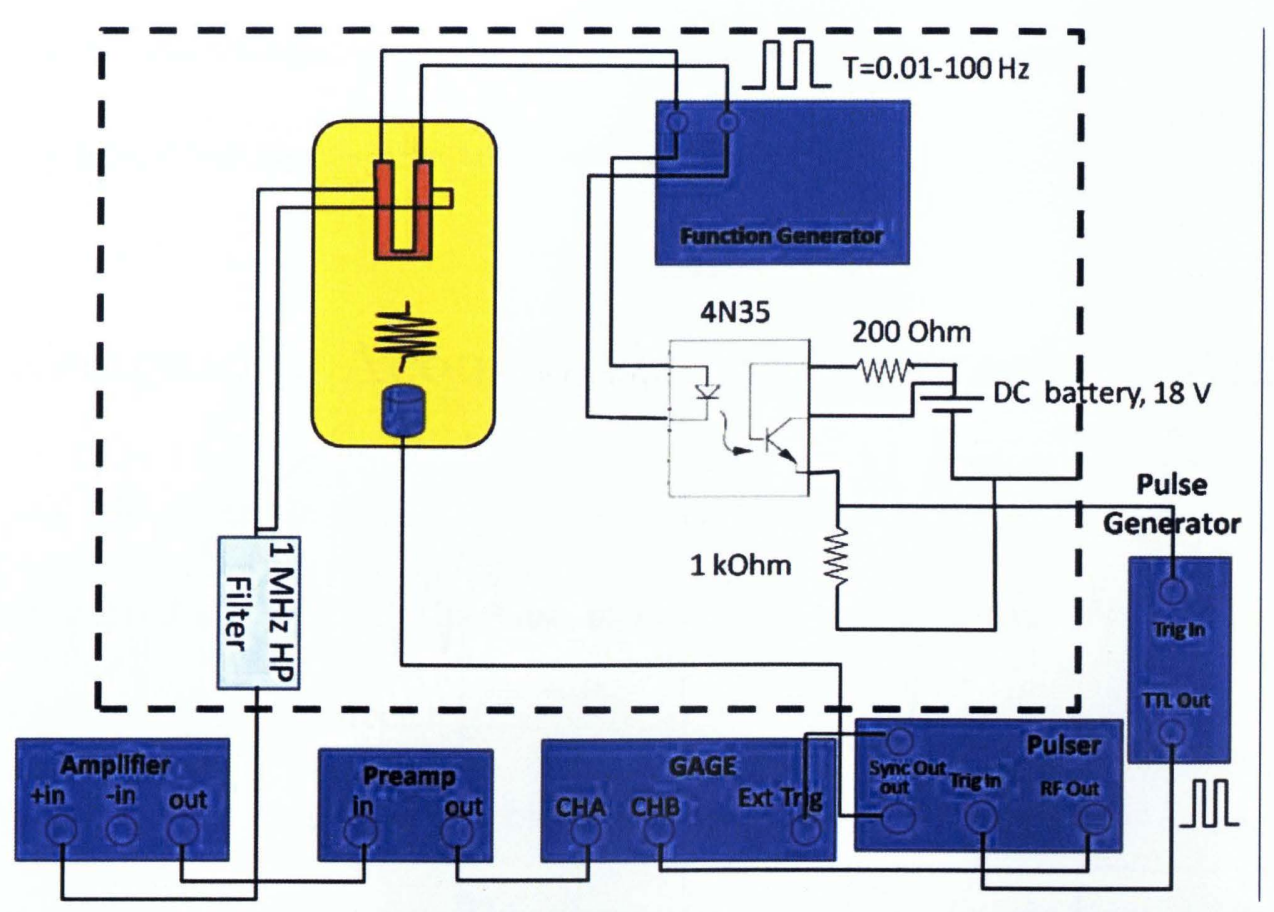

Figure 3.12: An illustration of the experimental setup to study the effect of the frequency of the applied $\mathrm{AC}$ voltage on the electrode signal.

optoisolator. During half the period of the applied AC voltage, the LED turned on making the transistor's base conductive for that period of time. Two DC batteries were applied in series with $200 \Omega$ resistor between the base and collector. The output voltage was detected using a $1 k \Omega$ resistor in series with the collector and emitter. This voltage was then applied to trigger the pulse generator. The pulse generator in turn generated square pulses separated by $250 \mu \mathrm{s}$. These pulses drove the pulser to produce high voltage bursts for the $1 \mathrm{MHz}$ transducer. The electrode signal from the pork sample was filtered and double-stage-amplified as before. The pulse-echo signals were acquired simultaneously with each scan and used to verify the position of the electrode signals. The data are shown on Fig. 3.13.

It can be seen from Fig. 3.13 that the electrode signal had a peak-to-peak amplitude of $0.4 \mathrm{mV}$ with fluctuations of $\pm 0.2 \mathrm{mV}$, which is exactly the noise level. Thus, the electrode 

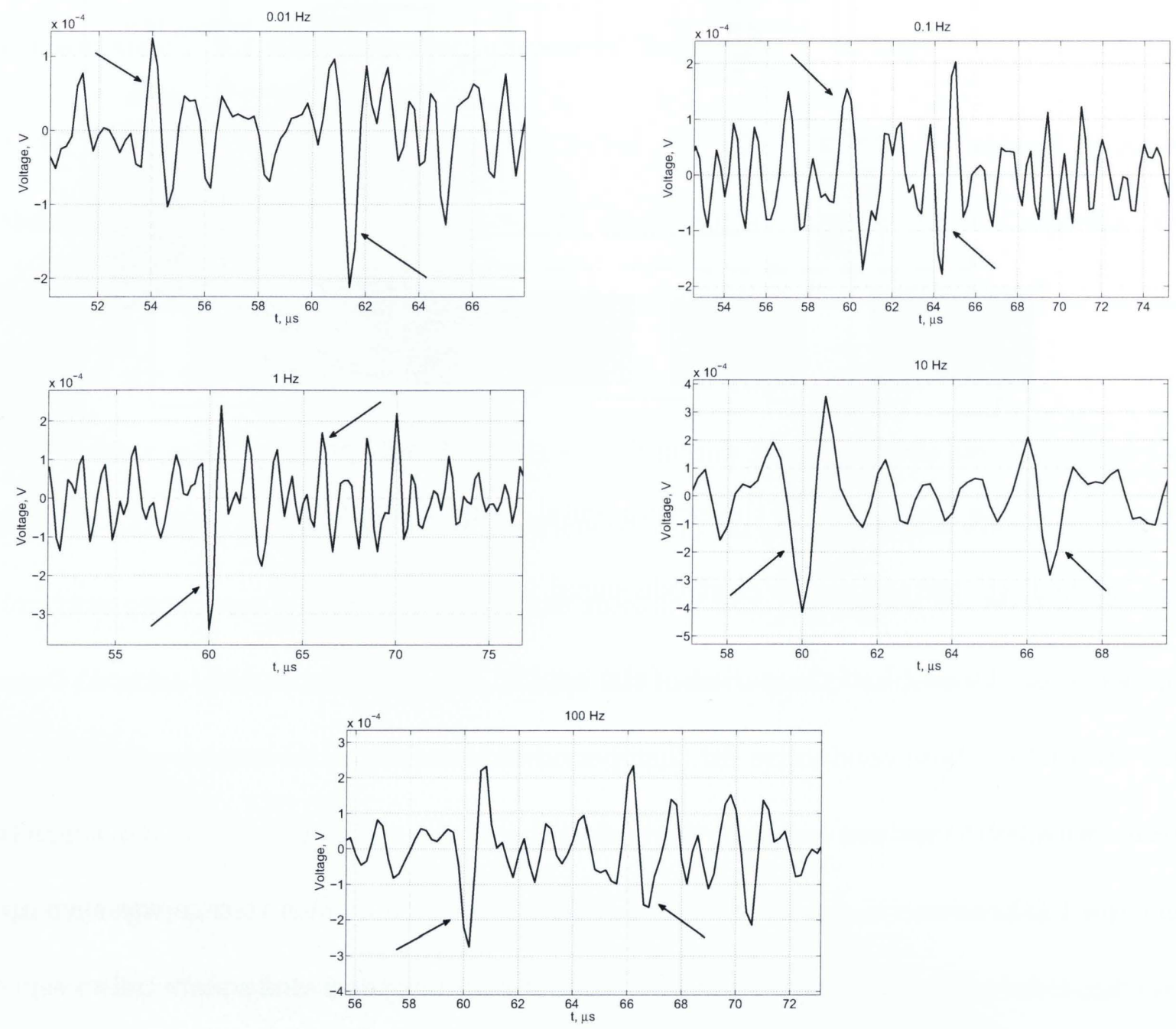

Figure 3.13: Experiments to illustrate the behavior of the electrode signal with frequency. Arrows mark the signal from the front and the rear interface between the oil and the sample. 
signal showed no correlation with the dispersion of the dielectric constant in tissue, being constant in a frequency range covering 5 orders of magnitude.

\subsection{Discussion. Acousto-electric interaction signals}

The fact that our electrode signal depends on the polarity of the applied voltage and does not noticeably decrease with the increase of frequency suggests that its source is, to most extent, the acousto-electric effect.

Acoustic pressure propagating in a biologic fluid (or electrolyte solution) or tissue induces a change in electrical conductivity. This phenomenon, acoustoelectric interaction (AE), was first described by Fox et al. in 1946 [43]. Jossinet et al. provided a more thorough analysis of the acoustoelectric effect in a binary electrolyte solution and performed experiments in isotonic saline $[44,45]$. Their derivation is based on the adiabatic nature of sound propagation.

Jossinet et al. [44] have derived in details the relationship between the impedance change in a medium and the propagating acoustic pressure. In it's simplified form, it can be expressed as

$$
\Delta \sigma=k \sigma_{0} \Delta P,
$$

where $\sigma_{0}$ is a conductivity of the medium, $\Delta P$ is a pressure change, $\Delta \sigma$ is a conductivity change, $k\left(\mathrm{~Pa}^{-1}\right)$ is the interaction coefficient of the medium. For a binary electrolyte solution, $k$ depends on the thermodynamic properties of the medium, as well as charge and mobility of each type of ion in the solution [45]. When acoustic pressure $P$ travels across a 
uniform conducting medium carrying current $i$, the change in conductivity leads to a voltage modulation $V(t)$ between two recording electrodes with resistance $R_{0}$ between them. Factor $k$ in study by Jossinet et al [44] varies from $95.2 \cdot 10^{-11} P a^{-1}$ for the solution of $\mathrm{KCl}$ to $98.5 \cdot 10^{-11} \mathrm{~Pa}^{-11}$ for a solution of $\mathrm{CaCl}_{2}$.

From Eq. 3.13 and 3.14, an AE voltage signal for our sample can be estimated. Lets assume that a $\Pi$-shaped sample of muscle tissue has a conductivity of $\sigma_{0}=0.08 \mathrm{Sm} \cdot \mathrm{m}$, with the distance between the electrodes $15 \mathrm{~cm}$ and cross section of $1 \mathrm{~cm}$. From Eq. 3.14,

$$
\Delta V_{m}=\int_{\Omega} \mathbf{J}_{\mathrm{ab}} \cdot \Delta \mathbf{E}_{1} \mathrm{dr} .
$$

From this equation, it can be seen that the change in the direction of the electric field $\mathbf{E}_{\mathbf{1}}$ will cause the change in the sign of $d V_{m}$, as follows from the dot product under the integral. Also, the following holds true when a conductivity changes in a volume confined by ultrasound:

$$
\Delta J=\Delta \sigma E=\sigma \Delta E
$$

and therefore,

$$
\Delta E=\frac{\Delta \sigma}{\sigma} E
$$

Now, for $10 \mathrm{~V}$ of applied voltage, $E=67 \mathrm{~V} / \mathrm{m}$. Next, $\frac{\Delta \sigma}{\sigma}=k d P=10^{-3}$. Assuming the particle velocity $1 \mathrm{~m} / \mathrm{s}$, density of muscle tissue $1.06 \times 10^{3} \mathrm{~kg} / \mathrm{m}^{3}, k=97 \times 10^{-11} \mathrm{~Pa}^{-1}$ and the speed of sound in tissue $1500 \mathrm{~m} / \mathrm{s}$, we get $d P=1.06 \mathrm{MPa}$ and, $\Delta E=67 \mathrm{mV} / \mathrm{m}$. As $\Delta E$ applies only to the volume affected by the ultrasonic pulse, which is the cylinder of 2 mm diameter and $\lambda / 2=0.75 \mathrm{~mm}, \Delta V=67 \mathrm{mV} \cdot \frac{1 \mathrm{~A}}{1 \mathrm{~cm}^{2}} \cdot 2.4 \cdot 10^{-9} \mathrm{~m}^{3}=1.6 \times 10^{-6} \mathrm{~V}$. In case 
of two-stage 6000 times amplification, we can expect the peak-to-peak voltage of $9.6 \mathrm{mV}$, which agrees with our results within an order of magnitude. The difference can be explained as follows. When it comes to the boundaries between the oil and the tissue, if the ultrasonic pulse is not incident normally on the boundary, the signal will have different phase across the beam diameter and, therefore, be weaker. Also, a characteristic value of the interaction coeffcient $k$ was used, as no data have been reported on its value for biological tissues.

The frequency of the acoustic field is not included in the interaction coefficient $k$ [45]. Therefore, one should not expect the acousto-electric interaction signal to change with frequency. 


\section{Chapter 4}

\section{Contrast provided by ultrasonic}

\section{vibration potentials}

\subsection{Experiments with heterogeneous samples}

With our experimental setup describe in 3.3.1, we were able to detect the ultrasonic vibration potential (UVP) from a muscle tissue. The ultrasonic vibration potential can be generated in an electrolyte, colloidal or biological tissue (refer to the Discussion section for more details).As such, we decided to perform more experiments to study the contrast between different tissues and find the signal value for our samples and extrapolate it to larger samples. we performed the experiments on the heterogeneous sample containing muscle and fat. The sample is shown on Fig. 4.1.

The sample contained two parallel stripes of fat and muscle tissue, each about $1 \mathrm{~cm}$ thick in the direction of the propagation of ultrasound. For better coupling and to bring the electrodes back from the focal zone, $10 \%$ saline agar gel was used. The electrodes were 


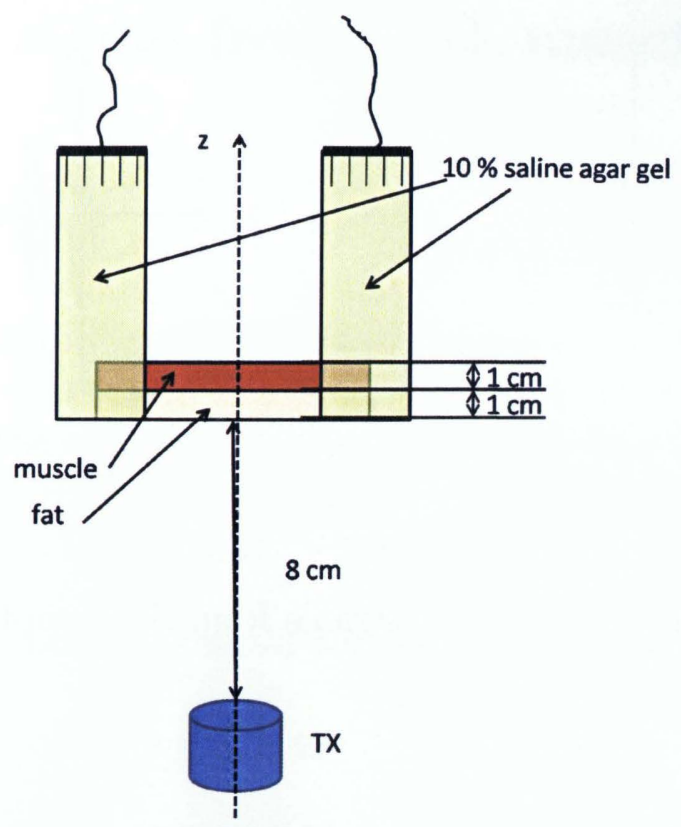

Figure 4.1: Heterogeneous sample used in our experiments and the position of the transducer. immersed in gel while solidifying as shown on Fig. 4.1. The front edge of the sample was 8 $\mathrm{cm}$ away from the $1 \mathrm{MHz}$ transducer. The ampification and filtering parameters are same as for the experiments described above, with a sampling rate of $5 \mathrm{MHz}$ and the averaging of $500 \times 10^{4}$. The results of these experiments are shown in Fig. 4.2.

First, from Fig. 4.2b, the position of the sample and interfaces can be determined. The

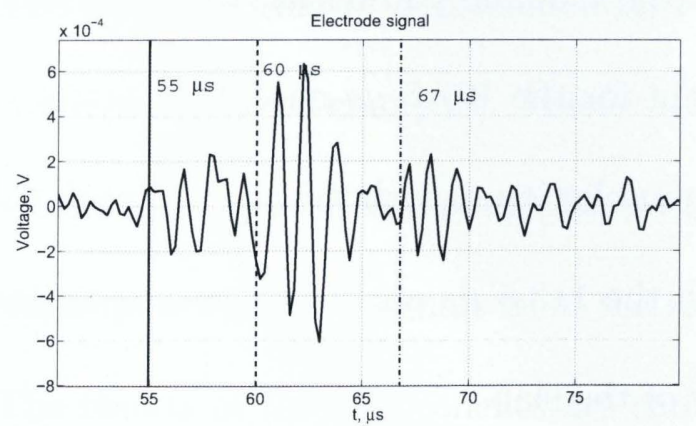

a

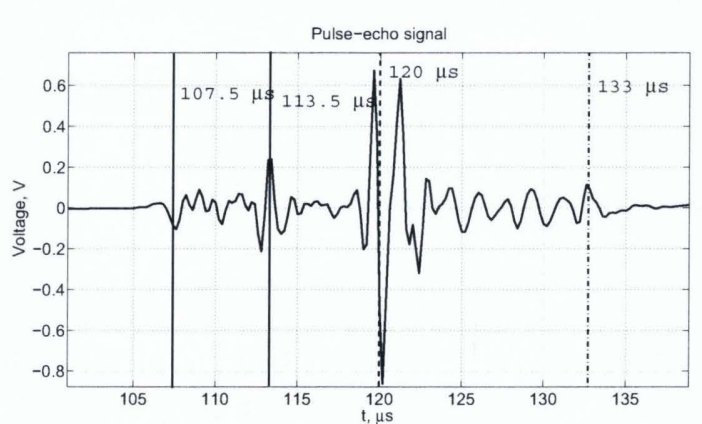

$\mathrm{b}$

Figure 4.2: Electrode (a) and acoustic (b) signals from the sample shown on Fig. 4.1. 


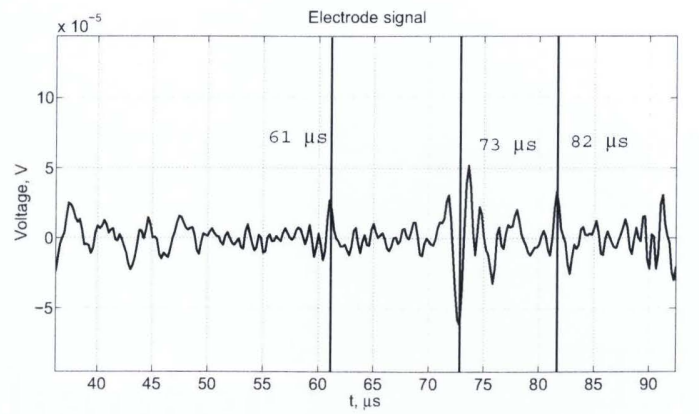

a

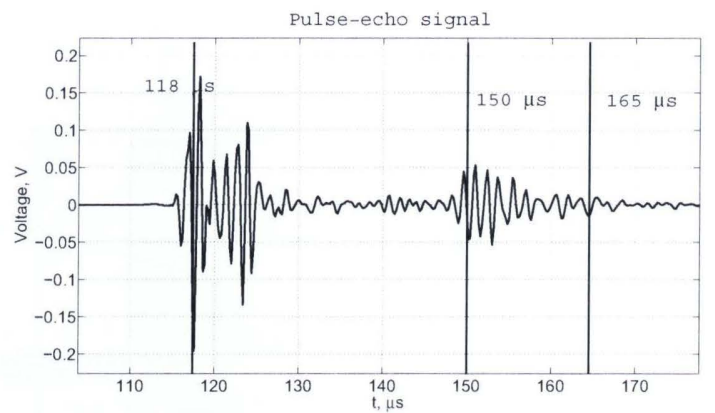

$\mathrm{b}$

Figure 4.3: Electrode (a) and acoustic (b) signals from the sample analogous to that shown on Fig. 4.1.

pulse-echo signal starts from 107.5 and ends at $133 \mu \mathrm{s}$, which corresponds to 8.1 and 10 $\mathrm{cm}$ away from the transducer. These distances agree with the size of the sample along the transducer's axis, which is $2 \mathrm{~cm}$. The pulse at $113.5 \mu \mathrm{s}$ corresponds to the distance of 8.5 $\mathrm{cm}$ away from transducer and may be due to the stripe of muscle tissues embedded in the fat. The interface between the fat and the muscle tissue appears to be $9 \mathrm{~cm}$ away from transducer $(120 \mu \mathrm{s})$, that is, the thickness of the fat slab is $0.9 \mathrm{~mm}$ along the transducer's axis. Next, one can compare the electrode signal (Fig. 4.2a) with the pulse-echo one. In the electrode signal, three pulses can be be identified: 55, 60 and $67 \mu s$, which corresponds to the 3 interfaces in the pulse-echo signal, except for the $107.5 \mu s$ one. The largest signal occured at the interface between the fat and the muscle tissue, with the peak-to-peak voltage of $1.2 \mathrm{mV}$. The burst at $55-57 \mu \mathrm{s}$ coincides with the $113.5 \mu \mathrm{s}$ on the pulse-echo signal and the $67 \mu s$ one corresponds to the rear boundary of the slab. 


\subsection{Electrode signal from pork muscle at frequency of}

\section{$10 \mathrm{MHz}$}

To see how the interaction of higher frequency acoustic waves with the internal structure of the muscle tissue affects the electrode signal, we acquired data from the pork muscle sample with a $10 \mathrm{MHz}$ transducer. The diagram of the experiments is shown on Fig. 4.4.

Fig. 4.4a shows the block diagram of the experimental setup. The sample was placed with the transducer in the oil tank inside the shielding box. The electrodes from the sample were connected to the BNC cable followed by the $46 \mathrm{~dB}$ preamplifier (Olympus, model 5670). For the second stage amplification, we used the built-in amplifier of the pulser with a gain of $20 \mathrm{~dB}, 1 \mathrm{MHz}$ high pass and full bandwidth low pass. The RF output of the pulser was consequently used for the electrode signal, and then for the pulse-echo signal to verify the position of the sample. The $1 \mathrm{MHz}$ high pass filter was used to eliminated the voltage drift that may the Gage card. The data were acquired at a sampling frequency of $50 \mathrm{MHz}$ with the averaging of $10^{6}$ for the electrode signal, and $10^{3}$ for the acoustic reflection signal.

The sample geometry is presented on Fig. $4.4 \mathrm{~b}$ and $4.4 \mathrm{c}$. The electrodes were $1 \mathrm{~cm}$ long and were positioned $6 \mathrm{~cm}$ apart. The focal length of the $10 \mathrm{MHz}$ transducer was $1.8 \mathrm{~cm}$. The electrodes were located far enough from the transducer to avoid causing disturbance within the time span when the signals from the sample are expected.

The results of the experiments are shown in Fig. 4.5. The signal in the upper row (a) contains 2 bursts at 16 and $21 \mu \mathrm{s}$. These bursts correspond to the front and rear boundaries of the muscle slab, as can be concluded from the corresponding pulse-echo signal (b) starting at around $33 \mu \mathrm{s}$. The reflection signal contains a lot of tails probably corresponding to multiple 


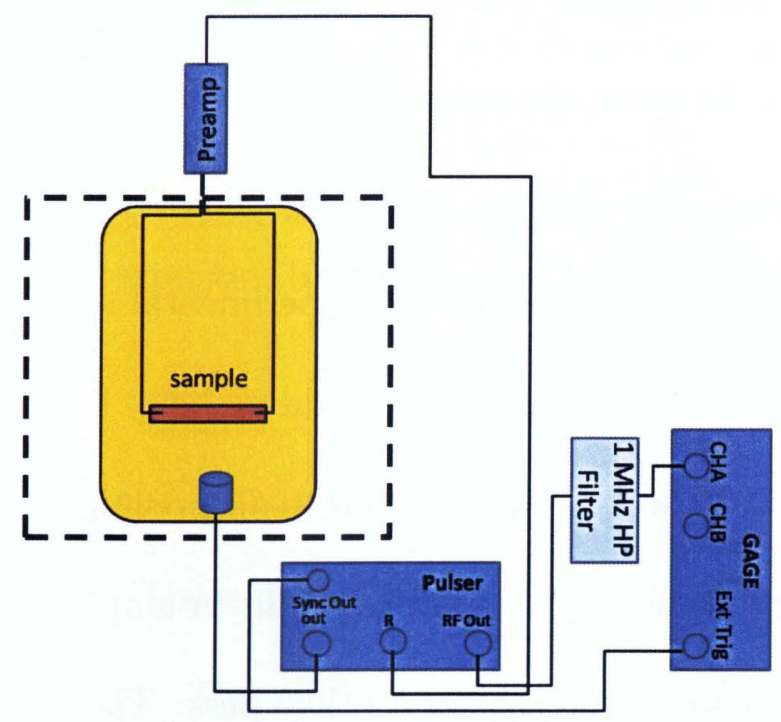

a

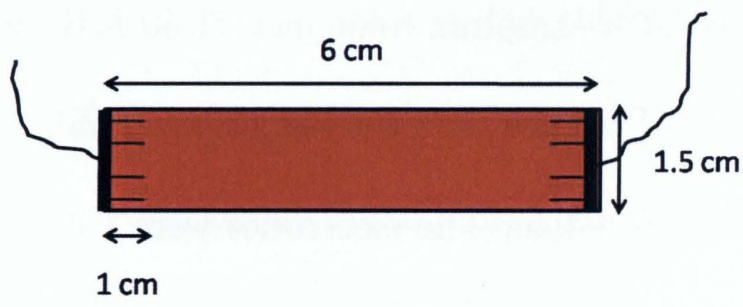

b

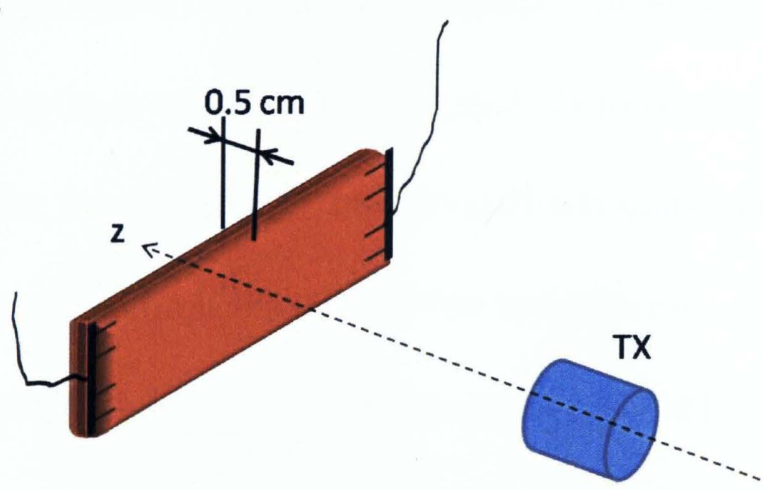

C

Figure 4.4: (a) Illustration of the experiments with the $10 \mathrm{MHz}$ transducer. (b) Dimensions of the sample in front view. (c) Dimensions of the sample in 3D view 


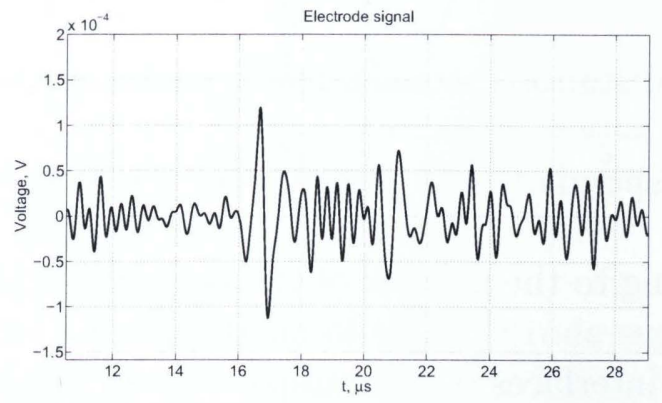

a

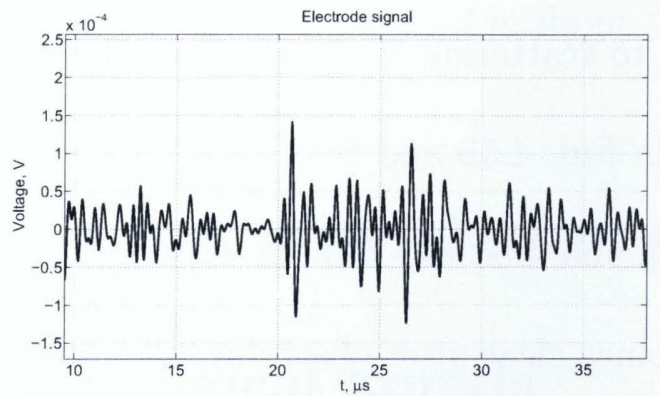

$\mathrm{C}$

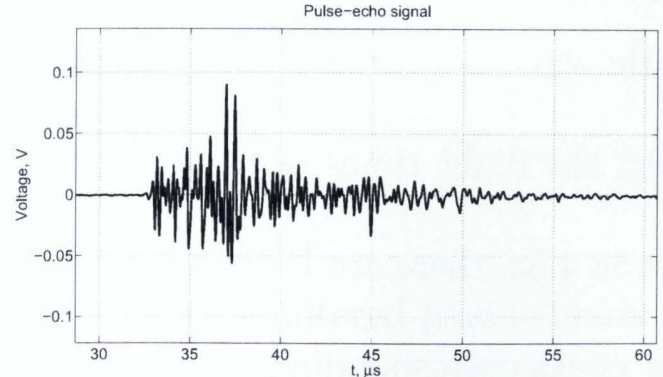

$\mathrm{b}$

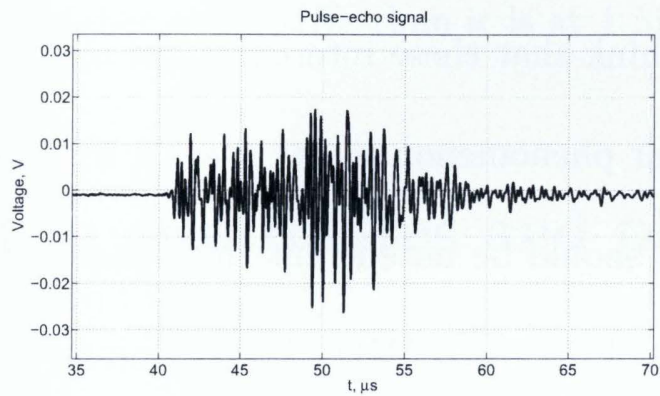

d

Figure 4.5: Results of the experiments with the $10 \mathrm{MHz}$ transducer. Each row corresponds to a particular flight time between the transducer and the sample. Left column contains are the uvp signals, at right column are the pulse-echo signals. 
reflections of the ultrasonic waves inside the sample. Thus, the position of the sample could only be verified from the start time of the pulse-echo signal as well as from the known sample width along the direction of the transducer's axis.

The lower row of Fig. 4.5 presents acquisitions for a different distance between the transducer and the sample. The UVP signal contains bursts at $21 \mu s$ and $26 \mu s$, while the acoustic signal starts at $41 \mu \mathrm{s}$. Thus, for both distances between the transducer and the sample, the flight times of the UVP signal corresponds to the positions of the sample. In Fig. $4.5 \mathrm{a} 4.5 \mathrm{c}$, there are UVP signals corresponding to the interior of the sample in addition to the peaks corresponding to the front and rear interfaces of the sample. For example, the signal on Fig. 4.5c has a larger peak-to-peak value, $0.125 \mathrm{mV}$, than the background, $0.1 \mathrm{mV}$. We think that these interior signal might be due to scattering from many small particle, a similar phenomenon to the speckle signal shown in Fig. 4.5b and Fig. 4.5d.

It should be noted that the signal in Fig. $4.5 \mathrm{a}$ and $4.5 \mathrm{c}$ was filtered within a frequency range of 0.1 to $10 \mathrm{MHz}$. The reason for that becomes clear after observing the spectrum of the UVP signal on Fig. 4.6. The spectrum contains the two bands at around $1 \mathrm{MHz}$ and 5 MHz. The reason that the $10 \mathrm{MHz}$ is not the central frequency is probably the absorption properties of the sunflower oil. Chanamai et al [46] obtained an empirical formula for the attenuation coefficient for a number of edible oils. The attenuation coefficient $\alpha$ is a power function of frequency:

$$
\alpha=A \cdot f^{n},
$$

where, for the sunflower oil, $A=5.68 \times 10-12$ and $n=1.85$. Thus, the attenuation 


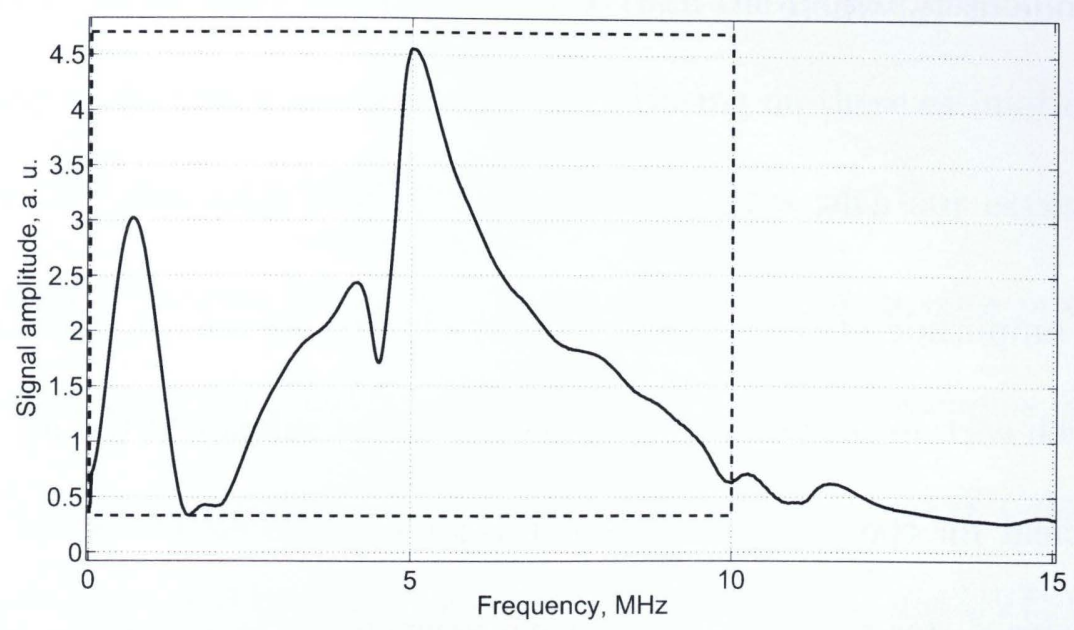

Figure 4.6: Spectrum of the electrode signal on Fig. 4.5a. The filtered part is marked with a dashed rectangle.

coefficient for the sunflower oil is about 200 times higher at $10 \mathrm{MHz}$ than it is at $1 \mathrm{MHz}$.

\subsection{Discussion. Debye effect in electrolytes and phys-}

\section{iological liquids}

P. Debye [47] did a thorough analysis of the movement of ions in an electrolyte with the ultrasonic pressure, with respect to the determination of the mass of electrolytic ions. He used the fact that different ions in an electrolyte have different masses and different friction coefficients with respect to the fluid they are diluted in. This leads to the different velocities of the charged particles in a pressure field and results in charge separation. Therefore, one can expect the potential gradient (field) in a solution related with this charge separation. Assuming plane wave propagating in a z-direction and the existence of only one component of the electric field, in the same z-direction, Debye showed that the potential will oscillate 
with an amplitude of $\Phi_{0}$, where

$$
\Phi_{0}=1.4 \times 10^{-7} a_{0} \frac{\Sigma\left(p_{i} \zeta_{i} M_{i} / \rho_{i}\right)}{\Sigma\left(p_{i} \zeta_{i}{ }^{2} M_{i} / \rho_{i}\right)} \text { volts. }
$$

Here, $a_{0}$ is the amplitude of the particle velocity (in the solvent), $p_{i}$ the number of ions of i-th type in which each molecule is broken down, $\zeta_{i}$ is the valency of the ith ion, $\rho_{i}$ is the the viscosity coefficient for the i-th ion. This potential was also estimated for the case of only two ions in a solvent (for example, $\mathrm{NaCl}$ ) with valencies $\zeta_{1}=+1$ and $\zeta_{2}=-1$ and for the peak particle velocity of $a_{0}=1 \mathrm{~cm} / \mathrm{s}$ to be of some $10^{-5} \mathrm{~V}$, which can be easily detected by ordinary amplification methods.

Later, several experimental evidences of the existence of the ultrasonic vibration potential (UVP) have been reported. In [48], electric signals from several electrolytes and pure liquids were observed. As to biological liquids, Zana et al [49] performed experiments with amniotic liquid at frequency far below the range of medical interest; however, UVP depends only weakly on the frequency. Hunter [50] doubted the origin of those electric signals in electrolytes, suggesting that they originate due to the capacitance between the liquid and the inserted probes. In our experiments, however, this was not the case, as the localization of the signal was in the good agreement with the flight times of the corresponding reflections in the pulse-echo signal.

The ultrasonic vibration potential has different signs for the different electrolytes and colloids, due to the different properties of the particles [47, 51, 52]. Beveridge et al [52] suggest that blood should have different UVP from the muscle tissue - $10 \mu V$ compared to less than $0.02 \mu \mathrm{V}$ (at $0.5 \mathrm{MHz}$ ), as its partially colloidal and ionic character would 
give a significantly larger electroacoustic voltage than the muscle tissue. No considerations regarding the uvp in fat tissue has been reported. Basing on these estimations, a 6000-times amplification should give a UVP of $0.12 \mathrm{mV}$, which agrees with our experiment within an order of magnitude. Samples in [52] had larger dimensions than ones in our experiments; namely, they were $2.5 \mathrm{~cm}$ thick and $5.1 \mathrm{~cm}$ in diameter. More experiments need to be carried out with the UVPs from biological tissues, due to the little amount of published data.

For the signals generated by both the impedance modulation and UVP, the peak-to-peak voltage was observed to be very low for a cross section of $1-2 \mathrm{~cm}^{2}$. If we expect a signal of $0.5 \mathrm{mV}$ for our samples after 6000 times amplification, a signal for a larger sample that has a $10 \mathrm{~cm} \times 10 \mathrm{~cm}$ cross section along the electrodes, would be 50-100 times smaller, which would be scaled down to $\mu V$ order. Therefore, highly sensitive systems should be developed to apply the UVP and AEI in medical diagnostics. It would be even more complicated to detect uvp from large samples of biological tissues. 


\section{Chapter 5}

\section{Overall conclusions and future work}

We investigated various techniques to image the electrical properties of biological tissues by combining ultrasound with electromagnetic field. The goal is to develop new imaging modalities that have both the high spatial resoluation as sonography and the contrast of electrical properties of tissues. Mainly, we propose Difference Frequency Magneto-AcoustoElectrical Tomography (DF-MAET) to image the electrical conductivity, and investigated ultrasound-induced magnetic dipole effect to image the permittivity. We also studied the impedance modulation by ultrasound and ultrasonic vibration potentials.

In Magneto-Acousto-Elecrical Tomography (MAET), we aimed to image the distribution of bioimpedance in a biological tissue. The main challenge in MAET is that signals were only observed at interfaces between regions with different conductivities. DF-MAET aims to obtain signal from the interior of the sample. To do so, we were able to apply the radiation force to MAET. High spatial resolution images were formed by scanning the focal point across gelatine samples at the modulation frequency of $1 \mathrm{KHz}$ with the resolution equal to the ultrasound beam diameter. The DF-MAET signal decreased exactly like the velocity of 
the radiation force-induced vibrations up to a frequency of $3.2 \mathrm{kHz}$. The increase at higher frequencies was accouted for the nonlinear acoustic emission from the piezo crystal.

In Chapter 3, we presented the theory and the preliminary experimental results to measure the permittivity of biological tissues. We have shown that a magnetic moment density can be induced in a polarized tissue by ultrasound. And, we derived the expression for the voltage signal from a pick-up coil. We designed several pick-up coils and tested their sensitivity. However, we did not obtain the signal from the pick-up coil predicted by the theory with our current detection system. We obtained signals from the electrodes attached to the samples. However, the signals detected with electrodes were independent of the frequency of voltage source applied to the samples. Therefore, these signals were not generated by magnetic diploes induced by the movement of polarized charges in the tissues. Their origin was found to be the impedance modulation by ultrasound. The theoretical estimation for the electrode signal was in a good agreement with the detected signal.

Finally, we examined UVP signals from a muscle sample. For the first time, we reported and compared the UVP and impedance modulation signals from the same pork muscle sample. We also investigated UVP from heterogeneous sample containing fat and muscle tissues. The experiments with $10 \mathrm{MHz}$ transducer revealed the UVP signals from the interior of the muscle tissue, features similar to the speckles in sonography.

\subsection{Future work}

DF-MAET in its present setup lacks axial resolution, which is limited by the length of the focal zone. One potential improvement to the experimental setup is to use two angularly- 
separated transducers. To reduce the nonlinear low-frequency acoustic emission from a piezo crystal, a double-element transducer would be helpful. Higher magnetic flux density need to be applied to achieve good SNR while bringing the ultrasound intensity below the safety limit.

The results on imaging the magnetic moment distribution with a pick-up coil are preliminary. More study can be done on the design of pick-up coils and the detection system, i.e. amplification system and reducing the noise level. The latter might include isolation of the source system from the detection system in a way similar to one described in Section 3.4 for the experiments with $\mathrm{AC}$ voltage source. In this way we would also achieve lower noise level and minimize the initial disturbance contaminating the data through the ground loop. This, in turn, would make it possible to increase the gain of our amplification system. Also, to test the proposed theory on the generation of magnetic moments in polarized biological samples by ultrasound, it would be helpful to use better-controlled samples, such as, a water/oil emulsion. In fact, when it comes to biological tissues, the mechanism behind huge values of electrical permittivity is still not completely known; therefore, more studies on this topic are necessary.

The UVP signals had not been studied extensively with the biological tissues, though a number of results have been reported on electrolytes and colloids. Much attention was paid to muscle tissue and blood $[51,53]$. Future work should include studying the UVP contrast in different tissues. Both impedance modulation and UVP techniques at present state have signal amplitudes insufficient for them to be applied in clinical medical imaging. More efforts are needed to improve the signal-to-noise-ratio in these techniques. 


\section{Appendix A}

Electrical, magnetic and acoustic properties of the media and materials used in experiments 
Medium/Material

$\mathrm{c}, \mathrm{m} / \mathrm{s} \quad z$, MRayl $\quad \mu_{r} \quad \sigma, S m \cdot m^{-1}$

$\epsilon_{r}$

$\begin{array}{cccccc}10 \% \text { saline agar gel } & 1594 & 1.7 & 1 & 0.1 & 80(1 \mathrm{MHz}) \\ \text { muscle } & 1580 & 0.170 & 1 & 0.08 & 6.6 \times 10^{6}(10 \mathrm{~Hz}) \\ \text { fat } & 1450 & 0.138 & 1 & 0.02-0.04 & 10^{7}(10 \mathrm{~Hz}) \\ \text { water } & 1480 & 0.148 & 1 & 0.05 & 80(1 \mathrm{MHz}) \\ \text { sunflower oil } & 1450 & 1.33 & 1 & 0 & 3 \\ \text { glass } & 5000 & 15.2 & 1 & 0 & 7\end{array}$

Table A.1: Main acoustic and electromagnetic parameters of the media and materials used in experiments $[2,3,4,5]$. 


\section{Appendix B}

\section{Publications and conference}

\section{presentations}

E. Renzhiglova, V. Ivantsiv and Y. Xu. Difference-Frequency Magneto-Acousto-Electrical Tomography. Submitted to IEEE Transactions on Ultrasonics, Ferroelectrics and Frequency Control.

Chapter 2 of this thesis was presented at the 2008 Conference of Canadian Association of Physicists (CAP). 


\section{Bibliography}

[1] S. Haider. Magneto-acousto-electrical tomography: a potential imaging method for current density and electrical impedance. Master's thesis, Ryerson University, 2008.

[2] E. P. Papadakis, editor. Ultrasonic instruments and devices: reference for modern instrumentation, techniques and technology. Academic Press, New Holland, 1999.

[3] D. Miklavcic, N. Pavselj, and F. Hart. Electric properties of tissues. John Wiley and Sons, Inc., 2006.

[4] D. Formica and S. Silvestry. Biological effects of exposure to magnetic resonance imaging: an overview. Bio Medical Engineering Online, 3(11), 2000.

[5] H. Lizhi, K. Toyoda, and I. Thara. Dielectric properties of edible oils and fatty acids as a function of frequency, temperature, moisture and composition. Bio Medical Engineering Online, 88(2), 2008.

[6] D. Holder. Electrical impedance tomography: methods, history, and applications. IOP, London, 2005.

[7] R. Chaoshi, W. Huiyan, An. Yuan, and L. Guojing. Development of electrical bioimpedance technology in the future. In Proceedings of the 20th Annual Interna- 
tional Conference of the IEEE Engineering in Medicine and Biology Society, volume 20, page 10521054, 1998.

[8] H. Griffiths. Magnetic induction tomography. Measurement Science Technology, 12(8):1126-1131, 2001.

[9] J. Jossinet. Variability of impedivity in normal and pathological breast tissue. Medical and Biological Engineering and Computation, 34:346-350, 1996.

[10] H. Griffiths. Magnetic induction tomography. Measurement Science Technology, 12(8):1126-1131, 2001.

[11] A. Korjenevshy, V. Cherepenin, and S. Sapetsky. Magnetic induction tomography: experimental realization. Physiological Measurement, 21(1):89-94, 2000.

[12] J. Jossinet. Elementary electrodynamics. Technology and Health Care, 16:465-474, 2008.

[13] C. Gabriel. Dielectric Properties of Biological Materials. Taylor Francis Group, LLC, 2006.

[14] R. Pethig and D. B. Kell. The passive electrical properties of biological systems: their significance in physiology, biophysics and biotechnology. Physics in Medicine and Biology, 32(8):933-970, 1987.

[15] E. Premkumar and R. Sundararajan. A simulation study of the electrical of a biological cell. 63:297-307, 2005. 
[16] P. Aberg, I. Nicander, J. Hansson, P. Geladi, U. Holgren, and S. Ollmar. Skin cancer identification using multifrequency electrical impedance - a potential screening tool. IEEE TRANSACTIONS ON BIOMEDICAL ENGINEERING, 51(12):2097-2102, 2004.

[17] S. R. Smith, K. R. Foster, and G. L. Wolf. Dielectric properties of vx-2 carcinoma versus normal liver tissue. IEEE TRANSACTIONS ON BIOMEDICAL ENGINEERING, BME-33(5):522-524, 1986.

[18] M. Lazebnik, D. Popovic, McCartney L., C. B. Watkins, M. J. Lindstrom, J. Harter, S. Sewall, T. Ogivlie, A. Magliocco, T. M. Breslin, W. Temple, D. Mew, J. H. Booske, M. Okoniewski, and S. Hagness. Dielectric properties of vx-2 carcinoma versus normal liver tissue. Physics in Medicine and Biology, 52:6093-6115, 2007.

[19] A. J. Surowiez, S. S Stuchly, J. R. Barr, J. H. Swarup, A. Oske, M. Okoniewski, and S. Hagness. Dielectric properties of vx-2 carcinoma versus normal liver tissue. Physics in Medicine and Biology, 52:6093-6115, 2007.

[20] B. H. Brown and A. D. Seager. The sheffield data collection system. Clinical Physics and Physiological Measurement.

[21] R. Halter, A. Hatrov, and K. D. Paulsen. High frequency eit for breast imaging. In Conference in Biomedical Applied Electrical Impedance Tomography, volume 6th, 2005.

[22] R. H. Bayford. Bioimpedance tomography (electrical impedance tomography). Annual REview of Biomedical Engineering.

[23] A. Korjenevsky, V. Cherepenin, and S. Sapetsky. Magnetic induction tomography: experimental realization. Physiological Measurement, 21(1):89-94, 2000. 
[24] P. Ola, L. Paivarinta, and E. Somersalo. An inverse boundary problem in electrodynamics. Duke mathematical journal, 70:617-653, 1993.

[25] Nuo Gao and Bin He. Noninvasive imaging of bioimpedance distribution by means of current reconstruction magnetic resonance electrical impedance tomography. IEEE TRANSACTIONS ON BIOMEDICAL ENGINEERING, 55(5):1530-1538, 2008.

[26] M Joy, G. Scott, and M. Henkelman. In vivo detection of applied electric currents by magnetic resonance imaging. Magnetic Resonance Imaging, 7:89-94, 1989.

[27] A. Montalibet, J. Jossinet, and A. Matias. Scanning electric conductivity gradients with ultrasonically-induced lorentz force. Ultrasonic Imaging, 23(2):117-132, 2001.

[28] A. Montalibet, J. Jossinet, A. Matias, and D. Cathignol. Electric current generated by ultrasonically induced lorentz force in biological media. Medical Biological Engineering Computing, 39(1):15-20, 2001.

[29] H. Wen. Feasibility of biomedical applications of hall effect imaging. Ultrasonic Imaging, 22(2):123-136, 2000 .

[30] H. Wen, J. Shah, and R. S. Balaban. Hall effect imaging. IEEE Transactions on Biomedical Engineering, 45(1):119-124, 1998.

[31] S Ballantine. Reciprocity in electromagnetic, mechanical, acoustical, and interconnected systems. Proc. IRE, 17:929-951, 1929. 
[32] S. Haider, A. Hrbek, and Yuan Xu. Magneto-acousto-electrical tomography: a potential method for imaging current density and electrical impedance. Physiological Measurement, 29:S41-S59, 2008.

[33] Richard C.S. Cobbold. Foundations of Biomedical Utrasound. Oxford University Press, Oxford, 1931.

[34] J. Bercoff, S. Chaffai, M. Tanter, and M. Fink. Ultrafast imaging of beamformed shear waves induced by the acoustic radiation force in soft tissues. In Proceedings of the 2002 IEEE Ultrasound Symposium, 2002.

[35] M. Fatemi and J. Greenleaf. Ultrasound-stimulated vibro-acoustic spectrography. Science, 280(5360):82-85, 1998.

[36] K. Nightingale, R. Nightingale, T. Hall, and G. Trahey. The use of radiation force induced tissue displacements to image stiffness. In 23rd International Symposium on Ultrasonic Imaging and Tissue Characterization, May 27-29 1998.

[37] S. Sarvazyan, O. Rudenko, S. Swanson, J. Fowlkes, and S. Emelianov. Shear wave elasticity imaging: A new ultrasound technology of medical diagnostics. Ultrasound Med. Biol., 24:1419-1435, 1998.

[38] K. Nightingale, M. Soo, R. Nightingale, and G. Trahey. Acoustic radiation force imaging: in vivo demonstration of clinical feasibility. Ultrasound Med. Biol., 28.

[39] G. Silva, A. Frery, and M Fatemi. Image formation in vibro-acoustography with depthof-field effects. Computerized Medical Imaging and Graphics, 30:321-327, 2006. 
[40] A. D. Hall. Review nonlinear effects in piezoelectric ceramics. Jornal of Materials Science, 36:4575 4601, 2001.

[41] Mark F. Hamilton and David T. Blackstock, editors. Nonlinear Acoustics. Academic Press, Toronto, 1998.

[42] E. Renzhiglova and Y. Xu. The possibility of imaging permittivity of biological tissues with ultrasound. In International Conference on Electrical Bioimpedance, 2010.

[43] F. Fox, K. Herzfeld, and G. Rock. The effect of ultrasonic waves on the conductivity of salt solutions. Physical Review, 70(5-6):329-339, 1946.

[44] J. Jossinet, B. Lavandier, and D. Cathignol. The phenomenology of acousto-electric interactin signals in aqueous solutions of electrolytes. Ultrasonics, 36:607-613, 1998.

[45] J. Jossinet, B. Lavandier, and D. Cathignol. Impedance modulation by pulsed ultrasound. Annals of the New York Academy of Sciences, 873:396-407, 2006.

[46] R. Chanamai and D. J. McClemens. Ultrasonic attenuation of edible oils. Journal of American Oil Chemistry Society, 75(10):1447-1448, 1998.

[47] P. Debye. A method for the determination of the mass of electrolytic ions. Journal of Physical Chemistry, 1:13-16, 1933.

[48] A. J. Rutgers and W. Rigole. Ultrasonic vibration potentials in colloidal solutions, in solutions of electrolytes and in pure liquids. Transactions of the Faraday Society, 54:139-143, 1957. 
[49] R. Zana and J. Lang. Interaction of ultrasound and amniotic liquid. Ultrasound in Medicine and Biology, 1:253-258, 1974.

[50] A. N. Hunter and T. B. Jones. The debye effect in electrolytes and colloids. Letters to the Editor, 1962.

[51] A. C. Beveridge, S. Wang, V. Gusev, and G. J. Diebold. Vibration potential imaging: Theory and preliminary results. In A. Oraevsky and L. V. Wang, editors, Proceedings of SPIE, volume 5320, 2004.

[52] A. C. Beveridge and G. J. Diebold. Tissue imaging using the ultrasonic vibration potential. In A. Oraevsky, editor, Proceedings of SPIE, volume 3916, 2000.

[53] A. Beveridge, S. Wang, and G. Diebold. Imaging based on the ultrasonic vibration potential. Applied Physics Letters, 85:5466-5468, 2004. 